\title{
4 Die Wirkung von Erzählungen
}

In der antiken Erzähltheorie werden v. a. zwei Wirkungen, die von Erzähltexten ausgehen, häufig diskutiert: die Unterhaltung und der Nutzen. Ihre prägnante Formulierung haben diese beiden Wirkziele in Horaz' Ars poetica in der Form erhalten, dass sie entweder einzeln oder zusammen verfolgt werden können, so dass die dritte Möglichkeit in der Kombination dieser beiden Wirkungen besteht: ${ }^{509}$

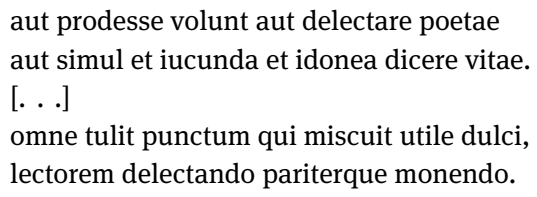

[Die Dichter wollen entweder nützen oder erfreuen oder zugleich das Angenehme und für das Leben Geeignete sagen. [. . .] Allgemeinen Beifall erntet derjenige, der das Nützliche mit dem Angenehmen vermischt (hat), indem er den Leser gleichermaßen erfreut und ermahnt.]

Diese beiden Wirkziele werden seit Homer und Hesiod mit der Dichtung bzw. mit Erzählungen verknüpft, wie die folgende Systematik belegen wird. Zwar wird die Unterhaltung / das Vergnügen / die Gemütsbewegung in der antiken Erzähltheorie häufig auf die Fiktion zurückgeführt und der Nutzen / die Belehrung auf eine wahre Darstellung (s. Kap. 4.1.1). Die folgende Systematik wird aber vor Augen führen, dass diese beiden Wirkungen ohne wesentlichen Unterschied für alle Erzählgattungen gelten, ohne dass die Faktualität bzw. Fiktionalität der Gattungen in dieser Hinsicht ein Differenzkriterium bildet. Zugleich wird herausgearbeitet, auf welche verschiedenen Elemente der Geschichte oder der Darstellung sich die Unterhaltung (Kap. 4.1) und der Nutzen / die Belehrung (Kap. 4.2) beziehen. In gleicher Weise werden auch die folgenden Wirkungen von Erzählungen präsentiert: die Überzeugung/Plausibilität (Kap. 4.3) und die Exemplifizierung (Kap. 4.4). ${ }^{510}$

509 Hor. ars $333 \mathrm{f}$. und $343 \mathrm{f}$.

510 Zur Wirkung der Dichtung vgl. Müller (2012, 165-246). Zu docere und delectare bei römischen Fachschriftstellern vgl. Paniagua Aguilar (2006). $\mathrm{Zu}$ den Wirkungen docere, delectare und movere in der Rhetorik und Poetik vgl. Adam (1971). 


\subsection{Unterhaltung/Vergnügen/Gemütsbewegung}

Das Ziel, die Rezipienten zu unterhalten (das Vergnügen und die Gemütsbewegung werden in diesem Zusammenhang häufig mehr oder minder als Synonyme genannt), wird durch mehrere Mittel erreicht, die sich zwar nicht immer trennscharf voneinander unterscheiden lassen, aber schwerpunktmäßig den in den folgenden Unterkapiteln genannten Kategorien zuordnen lassen, wobei die Auflistung nicht abschließend sein wird.

Die Tatsache, dass verschiedene Elemente der Geschichte und der Darstellung die unterhaltende Wirkung von Erzählungen hervorrufen, wird nicht nur durch die folgende Systematik deutlich, in der die verschiedenen Elemente einzeln präsentiert werden, sondern insbesondere auch durch Aristoteles' Reflexionen über die Wirkung von Erzählungen, wenngleich er insbesondere die Tragödie im Blick hat. Trotzdem gelten Aristoteles' Reflexionen über die Wirkung der Tragödie teilweise auch für das Epos, da Aristoteles so viele Gemeinsamkeiten zwischen den beiden Gattungen sieht, dass er zuerst die Tragödie und anschließend das Epos nach der im fünften Kapitel der Poetik geäußerten Programmatik behandelt, dass das Epos alle Teile aufweist, die auch der Tragödie zugrunde liegen, die Tragödie aber darüber hinaus eigene Teile und Spezifika aufweist, die nicht auf das Epos zutreffen. ${ }^{511}$

Folglich darf weder die tragödienspezifische noch die epenspezifische Wirkung als Ziel des Erzählens angesehen werden, sondern diejenige(n) Wirkung(en), die beide Gattungen verfolgen. Die tragödienspezifische Wirkung ist das Er-

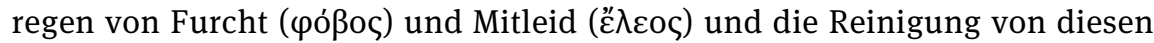
Affekten bzw. dieser Affekte. ${ }^{512}$ Die spezifische Wirkung des Epos nennt Aristoteles nicht explizit, obwohl er davon spricht, dass es sie gibt. ${ }^{513}$ Es lässt sich

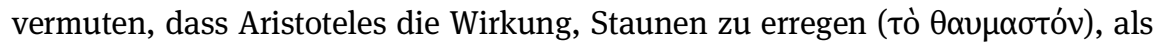
typisch für das Epos ansieht. Seine diesbezüglichen Äußerungen zeigen aber zugleich, dass auch die Tragödie diese Wirkung erzielt und nur ein gradueller

511 Vgl. Arist. Poet. 1449b9-20, v. a. 16-20. Daher beschränkt sich Aristoteles nach der Behandlung der Tragödie darauf, die spezifischen Unterschiede zwischen dem Epos und der Tragödie anzugeben (Kapitel 23 und 24, teilweise auch 25 und 26 der Poetik).

512 Vgl. Aristoteles' Definition der Tragödie (Arist. Poet. 1449b24-28). Vgl. auch Arist. Poet. 1452a1-4. Zur Kontroverse, ob der entsprechende Genetiv separativ oder objektiv aufzufassen ist, vgl. Schwinge (2012, 50 f., Fn. 11); Flashar (1956).

513 Vgl. Arist. Poet. 1459a20f. Vgl. auch seine Aussage über die Wirkung der Tragödie und des Epos (Arist. Poet. 1462b13f.). 
Unterschied in der Form besteht, dass das Epos mehr Möglichkeiten dazu hat, um dieses Ziel zu erreichen. ${ }^{514}$

Die grundlegende Wirkung der Erzählung ist nach Aristoteles zwar die Unterhaltung, wie die zuletzt genannte Passage nahelegt, da Aristoteles das Erstaunen als Form der Unterhaltung betrachtet, ${ }^{515}$ und wie mehrere andere Stellen deutlich machen, an denen er den Begriff der i்oovń als Oberbegriff für die Wirkungen der erzählenden Gattungen verwendet. ${ }^{516}$ Diese Stellen zeigen aber gleichzeitig, dass die Unterhaltung bei Aristoteles nicht in Opposition zum Nutzen bzw. zur kognitiven Wirkung der Dichtung zu sehen ist, sondern dass seine Dichtungskonzeption diese gewohnte Zweiteilung untergräbt.

Aristoteles verknüpft das Ziel, die Rezipienten zu erfreuen, sowohl mit der historischen als auch mit der fiktionalen Tragödie, wobei der letztere Typus eine Ausnahme darstellt, da das Grundgerüst der meisten Tragödien nach antiker Auffassung historisch ist. ${ }^{517}$ Auf welche Elemente der Geschichte oder Darstellung sich die erfreuende Wirkung bezieht, lässt sich nicht mit Sicherheit angeben. ${ }^{518}$ Verschiedene Beobachtungen sprechen aber dafür, dass sie aus allen qualitativen Teilen der Tragödie (s. Kap. 2.1.2.1) resultiert. Ein Hinweis hierauf ist, dass Aristoteles im sechsten Kapitel der Poetik die Lieddichtung

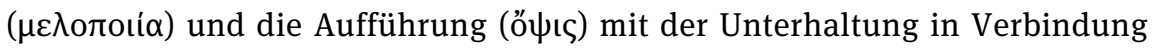
bringt. ${ }^{519}$ Ferner ist in Aristoteles' Definition der Tragödie die Rede von der

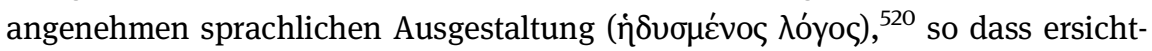
lich ist, dass auch dieser Teil zu einer erfreuenden Wirkung beiträgt. Außerdem lassen seine Aussagen über die spezifische Wirkung der Tragödie, die

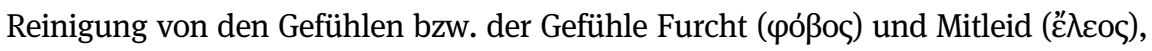

514 Vgl. Arist. Poet. 1460a11-17.

515 Aristoteles lehrt im 24. Kapitel der Poetik, dass dasjenige, was Staunen erregt, angenehm

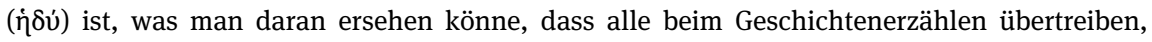

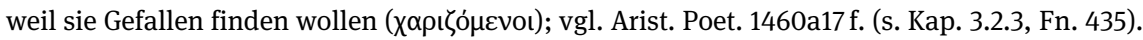
516 Vgl. Arist. Poet. 1453a35f. (über das schlechte Ende für die schlechten Tragödienfiguren und v. a. über das gute Ende für die guten Tragödienfiguren); 1453b8-14 (s. Fn. 521); 1459a21; 1462a16; 1462b13f.

517 Vgl. Arist. Poet. 1451b21-26 (s. Kap. 1.4.1.2).

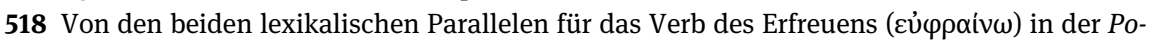
etik gewährt nur die eine Stelle Aufschluss über den allgemeinen Charakter dieser von Erzählungen, aber auch von anderen Künsten ausgehenden Wirkung, da Aristoteles das Erfreuen dort mit der Wirkung der Malerei (Arist. Poet. 1450b2) verbindet; an der anderen Stelle bezeichnet es ein Einzelereignis innerhalb des Ödipus-Mythos, nämlich dass ein Bote Ödipus eine erfreuliche Nachricht überbringen möchte (Arist. Poet. 1452a25).

519 Vgl. Arist. Poet. 1450b15-18. Vgl. auch Arist. Poet. 1462a14-17 (zum Vorrang der Tragödie gegenüber dem Epos).

520 Vgl. Arist. Poet. $1449 \mathrm{~b} 25$ und $28 \mathrm{f}$. 
Rückschlüsse auf das Ziel des Erfreuens bzw. Unterhaltens zu, da das zuletzt genannte Ziel als Oberbegriff ('̇ंovń) fungieren kann, der auch die zuerst genannten Affekte umfasst, wie das 14. Kapitel der Poetik zeigt:: ${ }^{51}$

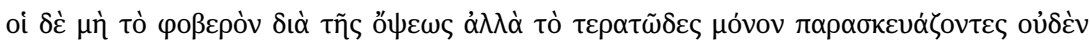

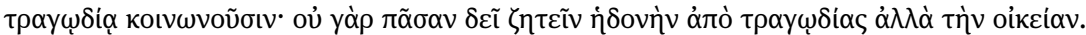

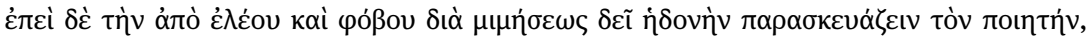

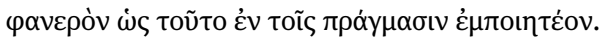

[Diejenigen [sc. Dichter], die durch die Inszenierung nicht dasjenige ausgestalten, was Furcht einflößt, sondern nur dasjenige, was wundersam ist, werden der Tragödie in keiner Weise gerecht. Denn man darf nicht jede Unterhaltung (†்ovท́) mit der Tragödie verfolgen, sondern nur die für sie charakteristische. Da der Dichter durch die Mimesis diejenige Unterhaltung, die aus Mitleid und Furcht resultiert, bewirken muss, ist klar, dass er dies in den Ereignissen tun muss.]

Da Aristoteles im 14. Kapitel der Poetik lehrt, dass Furcht und Mitleid auch durch die Inszenierung (ő $\psi \varsigma$ ) bewirkt werden können, dass diese Affekte am besten aber durch die Handlung hervorgerufen werden sollen, ${ }^{522}$ lässt sich annehmen, dass auch für die erfreuende Wirkung gilt, dass sie durch die Handlung oder die Aufführung, am besten aber durch alle qualitativen Teile der Tragödie in der Rangordnung, die Aristoteles ihnen zuweist (s. Kap. 2.1.2.1), also v. a. durch die Handlung und die Charaktere, bewirkt wird..$^{523}$

\subsubsection{Unterhaltung durch eine fiktionale Erzählung (vs. Nutzen/Belehrung durch einen wahren Bericht)}

Der Geograph Eratosthenes stellt die beiden Ziele, zu erfreuen und zu belehren, in der Form strikt gegenüber, dass die fiktionale Dichtung unterhält, aber nicht wie die historischen Werke - belehrt, wobei er mit $\psi v x \alpha y \omega y i ́ \alpha$ [Gemütsbewegung]

521 Arist. Poet. 1453b8-14.

522 Vgl. auch Arist. Poet. 1453b1-3.

523 Zur Tatsache, dass die Inszenierung von Aristoteles als Schmuck angesehen wird und

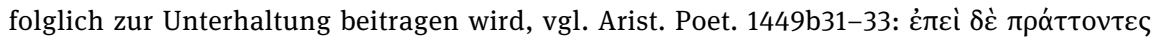

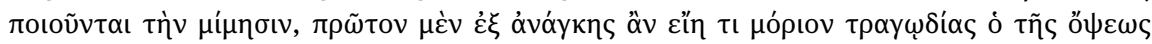

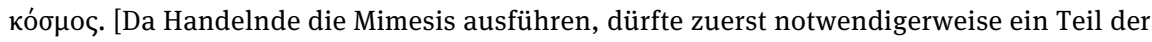
Tragödie der Schmuck der Aufführung sein.] Für die ergreifende Wirkung der Handlung,

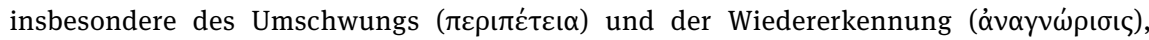
vgl. Arist. Poet. 1450a33-35. 
einen etwas allgemeineren Begriff als denjenigen des Erfreuens wählt (die relevanten Eratosthenes-Fragmente sind von Strabo überliefert): ${ }^{224}$

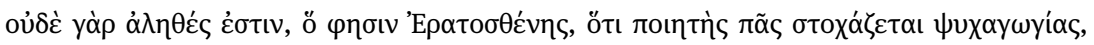
oủ $\delta เ \delta \alpha \sigma \kappa \alpha \lambda i ́ \alpha \varsigma$.

[Denn es ist auch nicht wahr, was Eratosthenes sagt, dass jeder Dichter es auf Gemütsbewegung, nicht auf Belehrung abgesehen hat.]

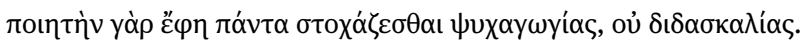

[Jeder Dichter, sagte er, habe es auf Gemütsbewegung, nicht auf Belehrung abgesehen.]

Diese Auffassung ist der Grund für Eratosthenes' Aufforderung, die Homerischen Epen nicht nach ihrem Sinn zu beurteilen und keine historische Kunde in ihnen zu suchen, sowie für sein ironisches Diktum, dass man erst dann herausfinden kann, wo Odysseus herumgeirrt ist, wenn man den Riemer gefunden hat, der den Sack der Winde genäht hat. ${ }^{525}$

Polybios zufolge ist insbesondere die Unterhaltung das Ziel von fiktionalen Darstellungen. Dies wird v. a. an derjenigen Stelle deutlich, an der Polybios (in

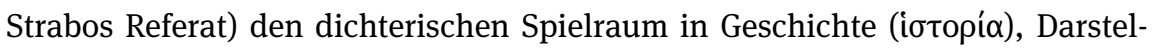

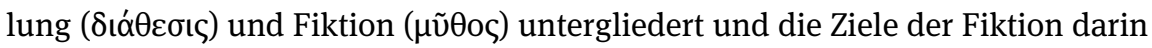
sieht, Vergnügen ( $\dot{\eta} \delta$ oví) zu bereiten und die Rezipienten in Staunen zu verset-

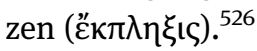

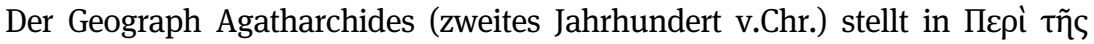

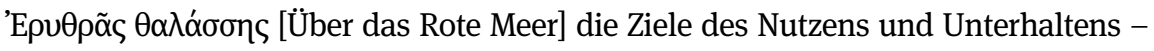

524 Strabo 1,1,10 und 1,2,3. Zum Eratosthenes-Zitat vgl. Eratosthenes, fr. I A 20 Berger $(1880,37)=$ fr. 2 Roller (2010, 41); vgl. auch den Kommentar von Roller (2010, 112-114).

525 Vgl. Strabo 1,2,15 = Polyb. 34,2,11 und Strabo 1,2,17 = Polyb. 34,4,4; Eratosthenes, fr. I A 16 Berger $(1880,36)=$ fr. 5 Roller (2010, 43); s. Kap. 1.4.1.3.

526 Vgl. Polyb. 34,4,1-4 = Strabo 1,2,17; s. Kap. 1.4.1.3. Aus einer anderen Stelle geht hervor,

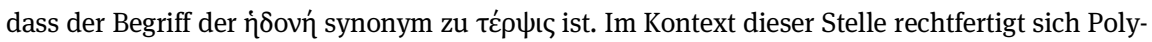
bios dafür, dass er die politische Form der Geschichtsschreibung verfolgt. Dabei assoziiert er die zurückgewiesene Gattung der Genealogie mit Fiktionen ( $\mu$ ṽ $\theta \mathrm{o})$. Diese verbindet Polybios mit der Wirkung, Vergnügen ( $\tau \dot{\rho} \rho \psi \iota)$ zu bereiten, wie ex negativo aus der folgenden Stelle hervorgeht, die ebenfalls zeigt, dass für die (pragmatische) Geschichtsschreibung insbesondere der

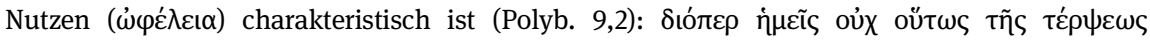

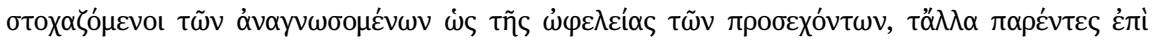

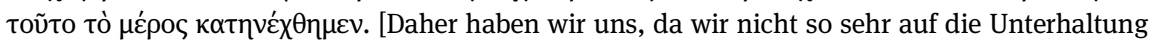
der Leser abzielen als vielmehr auf den Nutzen derjenigen, die sich dafür interessieren, diesem Teil zugewandt und das andere beiseitegelassen.] Neben der Genealogie weist Polybios (9,1f.) als zweite Art der Geschichtsschreibung die Darstellung von Kolonisationen, Städtegründungen und Verwandtschaftsverhältnissen zurück. Zum Nutzen der Geschichtsschreibung s. Kap. 4.2.1. 
ähnlich wie Polybios - nicht strikt gegenüber, sondern schwächt den Gegensatz im Sinne eines mehr oder weniger ab, indem er davon spricht, dass jeder Dichter mehr auf die Gemütsbewegung als auf die Wahrheit abzielt, wobei Agatharchides mit der $\psi v x \alpha y \omega y i ́ \alpha$ [Gemütsbewegung] - wie Eratosthenes - einen etwas allgemeineren Begriff als Unterhaltung verwendet. ${ }^{527}$

In Ciceros Prolog zum ersten Buch von De legibus werden ebenfalls die Unterhaltung und der Nutzen als Ziele von Erzählungen diskutiert, wenn Cicero erklärt, dass sich in der Geschichtsschreibung das meiste auf die Wirklichkeit und in der Dichtung, v. a. dem Epos, auf die Unterhaltung bezieht. ${ }^{528}$ Auffällig ist hierbei die Tatsache, dass zwar der wahre Bericht der Geschichtsschreibung mit dem Nutzen verknüpft wird, wenngleich dieser Begriff hier nicht gewählt wird, und die Dichtung aufgrund ihrer Fiktionalität mit der Unterhaltung in Verbindung gebracht wird, dass dieser Gegensatz aber nicht absolut ist. Vielmehr zeigt der Ausdruck pleraque [das meiste], dass es um ein mehr oder weniger geht und in beiden Gattungen beide Ziele verfolgt werden (können). ${ }^{529}$

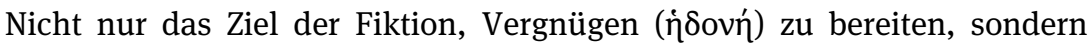
auch dasjenige, die Rezipienten in Staunen zu versetzen ( $\varepsilon^{\prime} \kappa \pi \lambda \eta \xi$ ıৎ), lässt sich bei Plutarch erkennen, der eine zum großen Teil fiktionale Aischylos-Tragödie im Blick hat, in der das Schicksal von Achill und Hektor behandelt wird: ${ }^{530}$

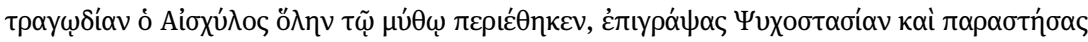

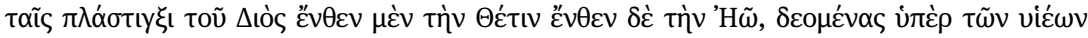

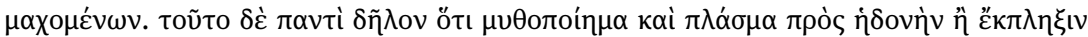

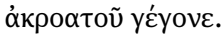

[Aischylos hat eine ganze Tragödie verfasst, in deren Mittelpunkt diese fiktive Geschichte

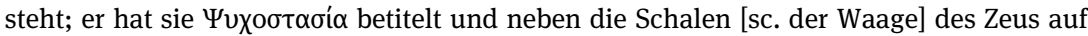
der einen Seite Thetis und auf der anderen Eos gestellt, die sich für ihre kämpfenden Söhne einsetzen. Jedem ist aber klar, dass dies eine ,mythische' Erfindung und Fiktion ist, die das Ziel hat, Freude und Erstaunen im Zuhörer hervorzurufen.]

527 Vgl. GGM I 8 Müller (1882, 117); s. Kap. 1.4.1.3.

528 Vgl. Cic. leg. 1,5; s. Kap. 1.4.2.4.

529 Im ersten Teil des Satzes (mit Bezug auf die Geschichtsschreibung) ergänzen die modernen Herausgeber ein Wort für „alles“ (z. B. omnia), wobei die Wahl und die Stellung des Wortes umstritten sind, und fassen pleraque [das meiste] nur als Subjekt des zweiten Teils des Satzes auf (mit Bezug auf die Dichtung). Diese Supplierung erweist sich aber zumindest als unnötig, da die Überlieferung einen guten Sinn ergibt; vgl. Feddern (2018, 460, Fn. 104).

530 Plut. mor. 17a. Zur हैk $\pi \lambda \eta \xi$ ıৎ vgl. Polyb. 2,56,11f. (s. Kap. 4.2.1); Polyb. 15,36,1-3 (s. Kap. 4.1.2.1); Polyb. 34,4,4 = Strabo 1,2,17 (s. Kap. 1.4.1.3). Vgl. auch Aristoteles’ Äußerun-

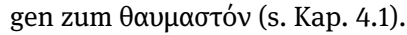


Die Beispiele für Reflexionen über die Unterhaltung durch eine fiktionale Erzählung ließen sich beliebig vermehren. ${ }^{531}$

\subsubsection{Unterhaltung durch eine faktuale Erzählung}

Der Begriff der faktualen Erzählung kann entweder als Oberbegriff für eine wahre und eine falsche Erzählung oder im Sinn einer wahren Erzählung verstanden werden. Hier wird dieser Terminus in der ersteren Bedeutung, also als faktualer Diskurs, verwendet.

Der Unterschied zwischen einer faktualen, aber falschen und einer fiktionalen Darstellung besteht darin, dass die fiktionale Darstellung durch eine soziale Praxis, die in der Moderne häufig „Fiktionsvertrag“ genannt wird, von der Verpflichtung entbunden ist, die Wahrheit mitzuteilen (s. Kap. 1.1.3.1). Der Verfasser einer falschen Darstellung zieht hingegen den Vorwurf des Irrtums, der Täuschung oder sogar der Lüge auf sich, da im faktualen Diskurs die Regel gilt, dass die Wahrheit mitgeteilt werden soll.

Der paradox anmutende Umstand, dass auch der Verfasser einer falschen Darstellung das Ziel verfolgt, die Rezipienten zu unterhalten (s. Kap. 4.1.2.2), erklärt sich dadurch, dass das Urteil, dass die entsprechende Darstellung falsch ist, nicht vom Verfasser, sondern von einem Kritiker stammt. In diesem Zusammenhang muss darauf hingewiesen werden, dass die Wahrheit häufig umstritten ist, ${ }^{532}$ etwa wenn der eine Historiker dem anderen Fehlinformationen vorwirft. In den folgenden Unterkapiteln kann es nicht darum gehen, zu entscheiden, wer Recht hat. Diese Frage können wir häufig aufgrund der Quellenlage nicht

$531 \mathrm{Vgl}$. z. B. Aug. soliloqu. 2,16,4: [. . .] et mimi et comoediae et multa poemata mendaciorum plena sunt, delectandi potius quam fallendi voluntate, et omnes fere qui iocantur, mentiuntur. [[. . . ] Auch die Mimen und Komödien und viele Gedichte sind voll von Unwahrheiten - mit der Absicht, eher zu unterhalten als zu täuschen -, und fast alle, die Scherze/Witze machen, sagen Unwahres.]

532 Vgl. Plutarch über die (dichterischen) Darstellungen des Todes und des Nachlebens (Plut.

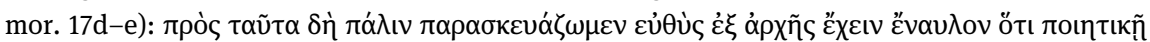

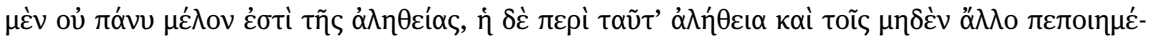

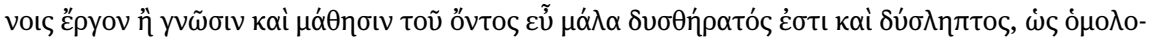

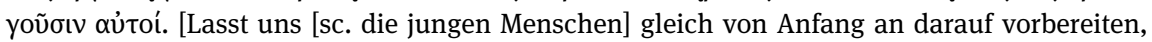
[sc. die Maxime] im Hinterkopf zu behalten, dass die Dichtung nicht auf die Wahrheit abzielt und dass die Wahrheit über diese Dinge auch für diejenigen, die keine andere Tätigkeit verfolgt haben als die Erkenntnis und das Erlernen des Seienden, sehr schwer zu erfassen und zu begreifen ist, wie sie selbst eingestehen.] Plutarch spricht im Kontext dieser Stelle über unabsichtlich falsche Äußerungen der Dichter, also über Irrtümer, nicht über die Fiktion. 
entscheiden, und sie muss in diesem Kontext nicht entschieden werden, da die systematische Einsicht im Mittelpunkt steht, dass sowohl falsche als auch wahre Erzählungen unterhalten. ${ }^{533}$

\subsubsection{Unterhaltung durch eine wahre Erzählung}

Das Ziel, die Rezipienten zu unterhalten, wird auch durch eine wahre Darstellung erreicht, die die Menschen deswegen erfreut, weil sie wissbegierig sind und/oder weil Ereignisse erzählt werden, die in irgendeiner Hinsicht mitteilungswürdig sind, weil sie über triviale Alltagsereignisse hinausgehen (in der modernen Erzähltheorie spricht man in Bezug auf faktuale Erzählungen vom Kriterium der tellability). ${ }^{534}$ Entsprechende Belege finden sich schon bei Homer und Hesiod. ${ }^{535}$

Im ersten Buch der Odyssee wird erzählt, dass der Dichter Phemios in Odysseus' Palast die leidvolle Rückkehr der Griechen aus Troja besingt. Während Penelope weint, nimmt Telemachos den Dichter mit den Argumenten in Schutz, dass sich die Menschen insbesondere an aktuellen Geschichten erfreuen und Odysseus nicht der einzige sei, der in Troja oder während der Heimfahrt gestorben sei: ${ }^{536}$

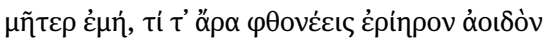

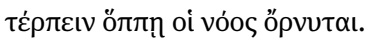

[Meine Mutter, warum nimmst du es dem zuverlässigen Sänger übel, dass er unterhält, wie auch immer der Sinn es ihm eingibt?]

An dieser Stelle bezieht sich die Wirkung des Erfreuens vornehmlich auf den Inhalt des Gesangs, der als faktual und teilweise falsch anzusehen ist, da Phemios sich irrt, wenn er davon ausgeht, dass Odysseus gestorben ist, da Odysseus in der Wirklichkeit des Epos auf den Meeren der Welt umherirrt. Innerhalb

533 Die folgenden Reflexionen über die unterhaltende Wirkung von wahren oder falschen Erzählungen werden nach der Maßgabe der einen oder anderen Rubrik zugeordnet, welche Perspektive der Autor einnimmt, welcher Standpunkt sich in einer Diskussion (eher) durchsetzt und/oder ob der wahre oder falsche Anteil überwiegt, soweit sich das entscheiden lässt.

534 Vgl. Zipfel $(2014,109)$.

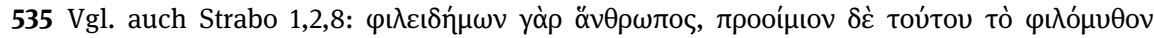
[Denn der Mensch hat Freude am Wissen, und die Vorstufe dazu ist die Freude an Fiktionen.]; Cic. de orat. 1,193 (in Bezug auf das Recht): mira quaedam in cognoscendo suavitas et delectatio [Eine doch bewundernswerte Annehmlichkeit und Unterhaltung liegt in der Erkenntnis.]

536 Hom. Od. 1,346f. Zum Kontext vgl. Hom. Od. 1,325-359. Zu denjenigen Szenen in der Odyssee, in denen die Sänger Demodokos und Phemios präsentiert werden, vgl. Bowie (1993, 14-17). 
des faktualen Diskurses sind wiederum zwei Aspekte besonders relevant für die unterhaltende Wirkung, nämlich zum einen die Neuigkeit der Informationen und zum anderen die Ruhmestaten von bedeutenden Männern. ${ }^{537}$ Möglicherweise tritt als weiterer Grund für die unterhaltende Wirkung des Dichters Phemios der Gesang hinzu (s. Kap. 4.1.6). ${ }^{538}$

An einer Stelle in Xenophons (zwischen 430 und 425 - nach 355 v.Chr.) Kyrupädie wird nicht nur über die unterhaltsame, sondern zugleich auch über die nützlich-belehrende Wirkung von wahren Erzählungen reflektiert, wenngleich dort nicht alle Gesprächspartner von der Wahrheit der Erzählungen überzeugt sind. An dieser Stelle schildert Xenophon ein gemeinsames Mahl des Kyros mit seinen Offizieren und berichtet davon, dass der Befehlshaber bei derartigen Zusammenkünften besonderen Wert auf das Erzählen von unterhaltsamen und zugleich zweckdienlichen Geschichten legte: ${ }^{539}$

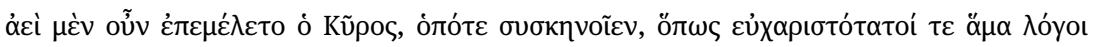

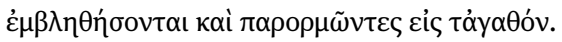

[Bei den gemeinsamen Mahlzeiten im Zelt trug Kyros stets dafür Sorge, dass Geschichten erzählt wurden, die zugleich sehr unterhaltsam waren und zum Guten anspornten.]

Es werden also die beiden Kategorien des delectare und des prodesse erwähnt. ${ }^{540}$ Bei den Geschichten handelt es sich um nahezu alltägliche Erzählungen, wie der Kontext deutlich macht. Im unmittelbaren Anschluss referiert Xenophon nämlich zwei lustige Anekdoten, die von Hystaspas und einem anderen, anonymen Offizier erzählt wurden und von schwer umgänglichen jungen Rekru-

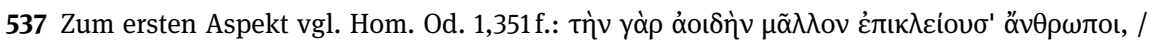

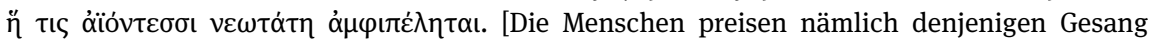
umso mehr, welcher auch immer als neuester den Zuhörern begegnet.] Zum zweiten Aspekt

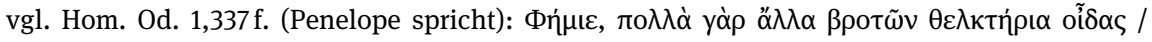

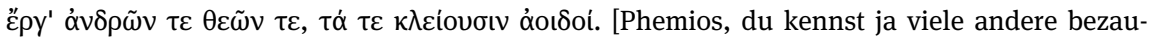
bernde Lieder von Sterblichen, Taten von Männern und Göttern, die die Sänger berühmt machen.]

538 In ähnlicher Weise wird in Hesiods Theogonie-Proömium (um 700 v.Chr.) die Wirkung des Gesangs beschrieben, wenngleich an dieser Stelle (Hes. Theog. 98-103) kein Wort benutzt wird, das „Unterhaltung“ bedeutet. Trotzdem lässt sich diese Wirkung darin erkennen, dass die von den Musen verliehene Gabe zur Dichtung die Menschen vom Leid ablenkt. Ähnlich wie im ersten Buch der Odyssee ist insbesondere der Aspekt der Ruhmestaten von bedeutenden Männern dafür verantwortlich, dass die faktuale Erzählung ihre unterhaltende Wirkung entfaltet. Hinzu kommt der Aspekt des Lobgesanges, der auch an einer anderen Stelle des Theogonie-Proömiums mit der Unterhaltung in Verbindung gebracht wird (s. Kap. 4.1.5).

539 Xen. Cyr. 2,2,1.

540 Vgl. Reichel $(1997,103)$. 
ten bei einem Mahl und bei einer Exerzierübung handeln. ${ }^{541}$ Zwar wird die Historizität der erzählten Anekdoten im weiteren Verlauf der Diskussion in Zweifel gezogen, wenn der Offizier Aglaïtadas Kyros fragt, ob er glaubt, dass die erzählten Geschichten wahr sind. ${ }^{542}$ Kyros erwidert aber hierauf mit der Gegenfrage, warum die Geschichten erlogen sein sollten, und auch der anonyme Offizier unterstützt den Standpunkt des Kyros. Die Diskussion endet, ohne dass die strittige Frage gelöst wird. ${ }^{543}$

Polybios benennt im Zusammenhang mit der Darstellung von Agathokles' Tod und seiner Kritik an anderen Historikern den Nutzen ( $\dot{\omega} \varphi \dot{\lambda} \lambda \varepsilon \mid \alpha)$ und die Unterhaltung ( $\tau \dot{\varepsilon} \rho \psi ı)$ ) als die beiden Ziele von Erzählungen: ${ }^{544}$

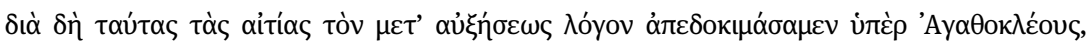

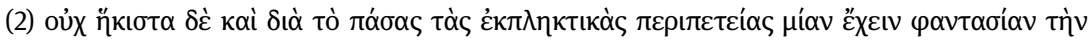

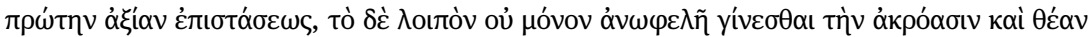

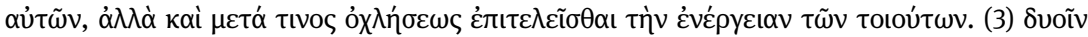
yà

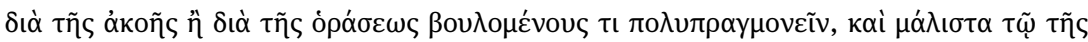

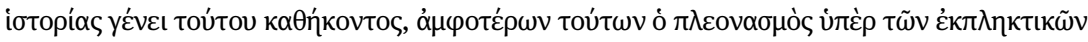

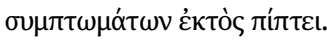

[Aus diesen Gründen haben wir jeden ausschmückenden Bericht über Agathokles vermieden, (2) nicht zuletzt auch deshalb, weil alle mitreißenden Wechselfälle des Schicksals nur beim ersten Lesen fesseln, später aber ihre audiovisuelle Rezeption nicht nur nutzlos ist, sondern mit einem gewissen Überdruss die Anschaulichkeit von derartigen Dingen erzeugt.

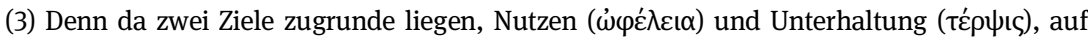
die diejenigen abzielen müssen, die sich auditiv oder visuell mit etwas beschäftigen möchten - und das gilt in besonderem Maße für die Gattung der Geschichtsschreibung -, verfehlt das Übermaß an erschütternden Ereignissen diese beiden [sc. Ziele].]

Wie diese Äußerungen deutlich machen, ist Polybios zufolge nicht der Nutzen das alleinige Ziel der Geschichtsschreibung und die Unterhaltung nur das Ziel der fiktionalen Gattungen, sondern die Kombination aus beidem (das Horazische delectare et prodesse) ist das Ziel aller erzählenden Gattungen und gilt insbesondere für die Geschichtsschreibung. ${ }^{545}$ Der Grund für die unterhaltende Wirkung der Geschichtsschreibung wird vorrangig in der wahren Erzählung lie-

541 Vgl. Xen. Cyr. 2,2,2-10.

542 Vgl. Xen. Cyr. 2,2,11.

543 Vgl. Xen. Cyr. 2,2,11-17.

544 Polyb. 15,36,1-3. Zu Polybios und zur sog. tragischen Geschichtsschreibung s. Kap. 3.2.1.3 und vgl. Pédech (1964). Zu Polybios' Darstellung von Agathokles' Tod vgl. Johstono (2017).

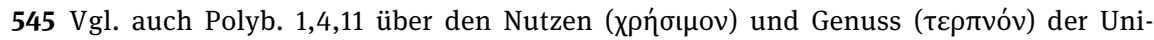
versalgeschichte. 
gen, wenngleich auch die Anschaulichkeit und somit ein Element der Darstellung (s. Kap. 4.1.3) zu dieser Wirkung beitragen wird. ${ }^{546}$

Der Gedanke, dass die Rezipienten einer wahren Erzählung (der Geschichtsschreibung) dadurch unterhalten werden, dass sie als wissbegierige Menschen aus einer wahren Darstellung etwas lernen, findet sich auch im fünften Buch von Ciceros De finibus, in dem der Sprecher den Gedanken entfaltet, dass es in der Natur des Menschen liegt, dass er bestimmte Tugenden hat und/oder entwickelt, die in ihm angelegt sind. ${ }^{547} \mathrm{Zu}$ den Tugenden, die der Mensch seit seiner Geburt hat und die deswegen nicht in seinem Willen stehen (virtutes non voluntariae), ${ }^{548}$ gehöre die Wissbegierde (hier umschrieben mit dem Ausdruck cognitionis amor et scientiae). ${ }^{549}$ In diesem Zusammenhang äußert der Sprecher die Ansicht, dass die Geschichtsschreibung den Menschen nicht nur nutzt, sondern sie auch erfreut, da sie ihnen Wissen vermittelt: ${ }^{550}$

ipsi enim quaeramus a nobis [. . . ] cognitiones quem ad modum nos moveant et quid historia delectet [. . . ]. nec vero sum nescius esse utilitatem in historia, non modo voluptatem.

[Fragen wir uns doch selbst, wie uns Erkenntnisse bewegen und welch erfreuende Wirkung die Geschichtsschreibung erzeugt. [. . .] Mir ist aber durchaus bewusst, dass Nützlichkeit in der Geschichtsschreibung liegt, nicht nur Vergnügen.]

Erst an zweiter Stelle wird nachgetragen, dass die Geschichtsschreibung auch nutzt, wobei das Wissen die Quelle sowohl der erfreuenden als auch der nützlichen Wirkung ist.

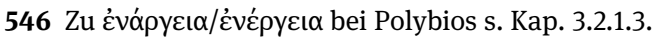

547 Vgl. Cic. fin. 5,34-58.

548 Vgl. Cic. fin. 5,36.

549 Vgl. Cic. fin. 5,48. Vgl. auch Cic. fin. 5,34 und 36 sowie 1,25. Zu den Tugenden, die in unserem Willen stehen (virtutes quae in voluntate positae sunt), gehören insbesondere die Kardinaltugenden Weisheit, Tapferkeit, Maßhalten und Gerechtigkeit (vgl. Cic. fin. 5,36).

550 Cic. fin. 5,51. In ähnlicher Weise erklärt sich die Ansicht des jüngeren Plinius, dass die Geschichtsschreibung Gefallen findet, auf welche Weise auch immer sie verfasst ist, durch die menschliche Wissbegierde (Plin. epist. 5,8,4): historia quoquo modo scripta delectat. sunt enim homines natura curiosi, et quamlibet nuda rerum cognitione capiuntur, ut qui sermunculis etiam fabellisque ducantur. [Die Geschichtsschreibung findet Gefallen, auf welche Weise auch immer sie verfasst ist. Die Menschen sind nämlich von Natur aus wissbegierig und werden durch die Erkenntnis der Dinge ergriffen, wie nackt diese auch sein mag; sie werden ja sogar von Gesprächen und Geschichtchen angezogen.] 


\subsubsection{Unterhaltung durch eine falsche Erzählung (vs. Nutzen/Belehrung durch einen wahren Bericht)}

Thukydides setzt unmittelbar vor dem Methodenkapitel seine eigene historiographische Darstellung von den Produkten der Dichter und Geschichtenerzähler mit dem Argument ab, dass sich diese nicht möglichst exakt an die Wahrheit halten, sondern nur das Ziel verfolgen, Freude bei den Zuhörern zu erregen: ${ }^{551}$

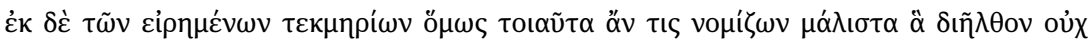

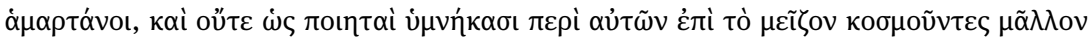

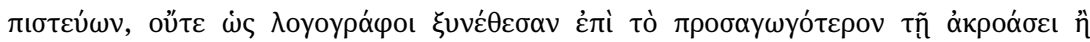

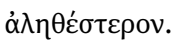

[Gleichwohl wird derjenige, der das, was ich dargelegt habe, aufgrund der angegebenen Beweise im Großen und Ganzen als solches [sc. Wahres] betrachtet, keinen Fehler machen. Er wird weder den Dichtern glauben, die es [sc. die Vergangenheit] in übertriebener Form verherrlicht haben, noch den (Prosa-)Schriftstellern, die mehr auf die Unterhaltung eines Publikums als auf die Wahrheit bedacht sind.]

Aus welchen - von Thukydides als falsch betrachteten - Elementen der Erzählungen der Dichter und Geschichtenerzähler die unterhaltsame Funktion resultiert, lässt sich kaum sagen. Möglicherweise verhalten sich aber Thukydides' Aussagen komplementär zueinander, dass die schwindelnden Schriftsteller die Vergangenheit in übertriebener Form verherrlicht haben und dass sie mehr auf die Unterhaltung eines Publikums als auf die Wahrheit bedacht sind: Die Unterhaltung ergibt sich aus den übertrieben enkomiastischen Zügen der entsprechenden Darstellungen (s. Kap. 4.1.5), wobei zur Unterhaltung möglicherweise auch das Erstaunen gehört. ${ }^{552}$

Die Unterhaltung durch eine falsche Darstellung steht bei Thukydides in Opposition zum Nutzen bzw. der Belehrung durch einen wahren Bericht, nämlich die eigene historische Darstellung: ${ }^{553}$

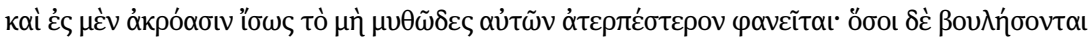

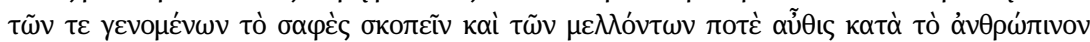

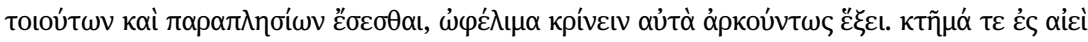

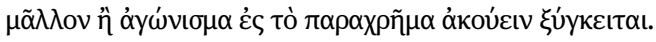

551 Thuk. 1,21,1. Zum Methodenkapitel s. Kap. 1.4.2.1.

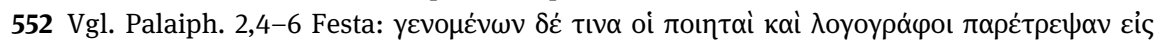

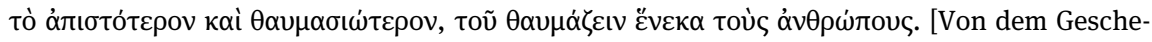
henen haben die Dichter und (Prosa-)Schriftsteller einigem einen recht unglaubwürdigen und wundersamen Charakter verliehen, damit die Menschen in Staunen geraten.]

553 Thuk. 1,22,4. 
[Für einen öffentlichen Vortrag ist meine Darstellung der Kriegsgeschehnisse wahrscheinlich zu wenig unterhaltsam, da ihr sagenhafte Züge fehlen. Wenn all diejenigen, die die Vergangenheit genau betrachten und die Zukunft [sc. vorhersehen] wollen, die sich gemäß der menschlichen Natur so und so ähnlich [sc. wie die Vergangenheit] verhält, meine Darstellung der Kriegsgeschehnisse als nützlich beurteilen werden, so wird das genügen. Als Besitz für alle Zeiten liegt sie vor, weniger als etwas, das dazu bestimmt ist, bei einem Vortragswettkampf nur für den Augenblick gehört zu werden.]

Dabei bezieht sich der Nutzen von Thukydides' Geschichtswerk nicht nur darauf, dass die Rezipienten historische Ereignisse der Vergangenheit erlernen, sondern auch in der Lage sind, daraus Schlussfolgerungen für die Zukunft zu ziehen. Nach Thukydides lassen sich am Peloponnesischen Krieg exemplarisch die Mechanismen aller Kriege studieren. ${ }^{554}$ Die sagenhaften Züge ( die Thukydides für sein Werk ablehnt, sind wahrscheinlich mehr oder minder deckungsgleich mit den zuvor genannten enkomiastischen Zügen der Erzählungen der Dichter und (Prosa-)Schriftsteller. ${ }^{555}$

\subsubsection{Darstellung (ästhetische Stilisierung)}

Eine weitere Möglichkeit, einer (fiktionalen oder faktualen) Erzählung eine unterhaltende Funktion zu verleihen, besteht in einer ansprechenden ästhetischen Stilisierung. ${ }^{556}$ So lobt Odysseus den Dichtersänger Demodokos im achten Buch der Odyssee auf eine Weise, die deutlich macht, dass er über die Genauigkeit und Anschaulichkeit des wahren Berichts vom Trojanischen Krieg spricht. ${ }^{557}$

554 Vgl. demgegenüber die konträre Dichtungskonzeption des Aristoteles, der das Allgemeine mit Blick auf die formale Handlungslogik bestimmt; s. Kap. 1.4.1.2.

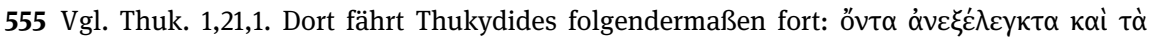

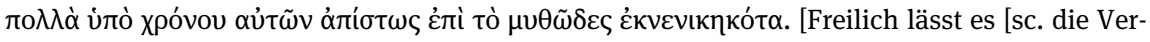
gangenheit] sich nicht mehr ermitteln, da das meiste, weil es vor langer Zeit geschah, bis zu einem unglaubwürdigen Grad sagenhafte Züge angenommen hat.] Flory (1990) zufolge trägt das Adjektiv $\mu v \theta \dot{\delta} \delta \eta \varsigma$ eine speziellere Bedeutung als $\mu \tilde{\theta} \theta$ o (,stories“, ,the storytelling element“), indem das Adjektiv patriotische Geschichten bezeichnet, die den Zuhörern Vergnügen bereiten. Scardino (2007, 415, Fn. 65) bemerkt aber zu Recht, dass diese Auffassung, die darauf beruht, dass Thukydides Herodots kriegsverherrlichende Geschichten kritisiert, zu einseitig ist.

$556 \mathrm{Vgl}$. Cic. part. 11: delectationem in exornatione [sc. orator sibi proponet] [Unterhaltung [sc. wird sich der Redner vornehmen] in der Ausschmückung]. Im Orator verknüpft Cicero das Ziel, das Publikum zu unterhalten, mit dem mittleren Stil; vgl. Cic. orat. 69. Vgl. auch Quint. inst. 8 pro. 7.

557 Vgl. Hom. Od. 8,488-491 (s. Kap. 3.2.1.3). Im Anschluss hieran verlangt Odysseus nach der Geschichte des hölzernen Pferdes; vgl. Hom. Od. 8,492-498. 
Das Ziel, durch eine ansprechende ästhetische Stilisierung dem Ziel der Unterhaltung zu dienen, verfolgt auch die Geschichtsschreibung. Entsprechende Reflexionen über die unterhaltsame Historiographie finden sich insbesondere im zweiten Buch von De oratore, in dem der Ciceronische Antonius über den schlichten Stil der frühen römischen Geschichtsschreibung spricht und durchblicken lässt, dass ein ansprechendes römisches Geschichtswerk von einem rhetorisch geschulten Literaten geschrieben werden müsste: ${ }^{558}$

erat enim historia nihil aliud nisi annalium confectio. [. . . ] (53) hanc similitudinem scribendi multi secuti sunt, qui sine ullis ornamentis monumenta solum temporum, hominum, locorum gestarumque rerum reliquerunt; itaque qualis apud Graecos Pherecydes, Hellanicus, Acusilas fuit aliique permulti, talis noster Cato et Pictor et Piso, qui neque tenent, quibus rebus ornetur oratio - modo enim huc ista sunt importata - et, dum intellegatur quid dicant, unam dicendi laudem putant esse brevitatem. (54) paulum se erexit et addidit maiorem historiae sonum vocis vir optimus, Crassi familiaris, Antipater; ceteri non exornatores rerum, sed tantum modo narratores fuerunt.

[Die Geschichtsschreibung war nämlich nichts anderes als das Anfertigen von Annalen. [. . . ] (53) Dieser Ähnlichkeit im Schreiben sind viele gefolgt, die ohne irgendwelche Schmuckmittel nur das Andenken an die Zeiten, Menschen, Orte und Ereignisse hinterlassen haben. Von welcher Art daher bei den Griechen Pherekydes, Hellanikus, Akusilas und sehr viele andere waren, so sind unser Cato, Pictor und Piso, die weder die Mittel haben, mit denen die Rede geschmückt wird - das ist nämlich gerade erst hierher importiert worden - und, solange verstanden wird, was sie sagen, die Kürze für den einzigen Vorzug des Redens halten. (54) Antipater, ein hervorragender Mann und Freund des Crassus, hat sich ein wenig erhoben und der Geschichtsschreibung einen größeren Ton der Stimme verliehen; die anderen haben ihre [sc. Darstellung der] historischen Ereignisse nicht stilistisch ausgeschmückt, sondern waren nur Erzähler [sc. von nackten Tatsachen].]

In diesem Zusammenhang äußert der Ciceronische Antonius die Ansicht, dass von Herodot eine unterhaltende Wirkung ausgeht, die aus seinem Stil resultiert: ${ }^{559}$

tanta est eloquentia [sc. Herodotus, qui princeps genus hoc ornavit], ut me quidem, quantum ego Graece scripta intellegere possum, magno opere delectet.

[Er besitzt eine so große Eloquenz [sc. Herodot, der als erster dieser Gattung Schmuck verliehen hat], dass er zumindest mich, soweit ich auf Griechisch verfasste Schriften verstehen kann, sehr erfreut.]

558 Cic. de orat. 2,52-54. Zur annalistischen Geschichtsschreibung vgl. das ähnliche Urteil Cic. fam. 5,12,5. Für den Gedanken, dass ein ansprechendes römisches Geschichtswerk noch (von Cicero) geschrieben werden muss, vgl. Cic. leg. 1,5. Zu Cicero und der Geschichtsschreibung s. Kap. 1.4.2.1, Kap. 1.4.2.4 und Kap. 2.1.3.3.

559 Cic. de orat. 2,55. Vgl. auch Cic. de orat. 2,59. 
Wie diese Stelle und ihr Kontext zeigen, wird Herodot, der Vater der Geschichtsschreibung, aber auch vieler fiktiver Geschichten, hier als Historiker erwähnt, der durch den Stil seines Werkes und nicht durch erfundene Geschichten die Rezipienten erfreut. 560

\subsubsection{Ungewöhnlich spannender Inhalt}

Auch und insbesondere ein spannender Inhalt, der z. B. in dem wechselhaften Schicksal des Hauptprotagonisten besteht, kann eine unterhaltsame Wirkung erzeugen. Dies erhellt aus Ciceros Lucceius-Brief, in dem Cicero seinen Freund dazu bewegen möchte, ein enkomiastisches Geschichtswerk über die Zeit von der Catilinarischen Verschwörung bis zu seiner Rückkehr aus dem Exil zu verfassen, und in dem das Vergnügen als eine Wirkung der Geschichtsschreibung bezeichnet wird: ${ }^{561}$

multam etiam casus nostri varietatem tibi in scribendo suppeditabunt plenam cuiusdam voluptatis, quae vehementer animos hominum in legendo tuo scripto retinere possit; nihil est enim aptius ad delectationem lectoris quam temporum varietates fortunaeque vicissitudines: quae etsi nobis optabiles in experiendo non fuerunt, in legendo tamen erunt iucundae, habet enim praeteriti doloris secura recordatio delectationem; (5) ceteris vero nulla perfunctis propria molestia, casus autem alienos sine ullo dolore intuentibus etiam ipsa misericordia est iucunda. quem enim nostrum ille moriens apud Mantineam Epaminondas non cum quadam miseratione delectat? [. . .] si vero exitu notabili concluduntur, expletur animus iucundissima lectionis voluptate.

[Auch unsere Schicksalsschläge werden dir beim Schreiben viel Abwechslung bereiten, die voll von einem gewissen Vergnügen ist, das die Menschen, wenn sie dein Werk lesen, stark im Bann halten kann. Denn für die Unterhaltung des Lesers ist nichts angemessener als abwechslungsreiche Zeiten und die Unwägbarkeiten des Schicksals. Auch wenn diese uns, als wir damit konfrontiert waren, nicht wünschenswert waren, so werden sie dennoch beim Lesen angenehm sein. Denn die sorgenlose Erinnerung an einen vergangenen Schmerz ist unterhaltsam. (5) Den übrigen aber, die keine eigene Beschwernis überstanden haben, die fremden Schicksalsschläge aber ohne jeden Schmerz betrachten, ist sogar das Mitleid selbst angenehm. Denn wen von uns erfreut jener bei Mantinea sterbende Epaminondas nicht mit einem gewissen Mitgefühl? [. . .] Wenn aber [sc. die Schicksalsschläge eines herausragenden Mannes] mit einem bemerkenswerten Tod abgeschlossen werden, wird man von einem äußerst angenehmen Lesegenuss erfüllt.]

$560 \mathrm{Zu}$ Herodot als dem Vater der Geschichtsschreibung, aber auch vieler fiktiver Geschichten, vgl. Cic. leg. 1,5 (s. Kap. 1.4.2.4).

561 Cic. fam. 5,12,4f. Zu diesem Brief vgl. Shackleton Bailey (1977, 318-322); Schorn (2019, 633-636); Rudd (1992). 
Es ist auffällig, dass Cicero in diesem Brief die unterhaltsame Wirkung insbesondere mit negativen Erlebnissen verknüpft, nämlich mit seinem Exil, und somit nur mit einem Ausschnitt desjenigen Zeitraumes, den Lucceius behandeln soll. ${ }^{562}$ Dieser Umstand erklärt sich aber durch die Tatsache, dass die Unterhaltung als Oberbegriff für die emotionale Wirkung eines Textes verwendet wird, zu der auch das Mitleid gehört. Dasselbe Phänomen lässt sich auch bei

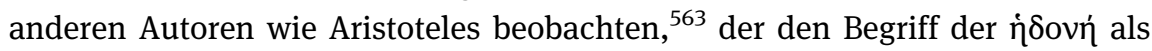
Oberbegriff für die Wirkungen der erzählenden Gattungen verwendet, wozu auch das Erzeugen von Furcht und Mitleid gehört (s. Kap. 4.1). ${ }^{564}$

\subsection{5 (Herrscher-)Lob, panegyrische Züge}

Ferner können auch das (Herrscher-)Lob und panegyrische Züge eine erfreuende Wirkung erzeugen - insbesondere, aber nicht ausschließlich für denjenigen, der gepriesen wird. In Hesiods Theogonie-Proömium ist die Rede davon, dass die Musen den Sinn des Zeus auf die folgende Weise erfreuen: ${ }^{565}$

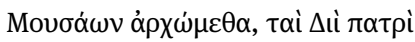

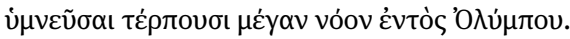

[Lasst uns mit den Musen beginnen, die mit ihrem Lobgesang den großen Sinn des Göttervaters Zeus erfreuen auf dem Olymp.]

Die Tatsache, dass die unterhaltende Wirkung von Erzählungen auch durch (Herrscher-)Lob erreicht wird, zeigt sich auch in der Rhetorik im Zusammen-

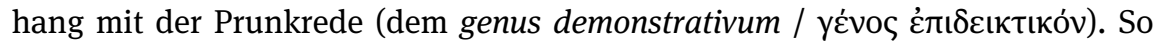
heißt es in Ciceros Partitiones oratoriae, dass der Adressat der Rede entweder erfreut wird oder etwas beschließt, wobei sich die erfreuende Wirkung auf das genus demonstrativum bezieht, wohingegen das Beschließen auf den Richter

562 Daher ist es kein Zufall, dass dieser Brief mit der sog. tragischen Geschichtsschreibung in Verbindung gebracht wurde; zur tragischen Geschichtsschreibung s. Kap. 3.2.1.3.

563 Vgl. auch Aug. conf. 3,2 (über Tragödienaufführungen): [. . .] dolor ipse est voluptas eius [[. . .] Der Schmerz selbst ist das Vergnügen von ihm [sc. des Zuschauers]].

564 Zur Unterhaltung, von der Cicero in diesem Brief spricht, wird sicherlich auch der enkomiastische Charakter des erhofften Geschichtswerks beitragen; vgl. insbesondere Cic. fam. 5,12,3. Zur Panegyrik als Quelle der Unterhaltung s. Kap. 4.1.5.

565 Hes. Theog. 36f. Vgl. auch V. 51, der nahezu identisch mit V. 37 ist. 


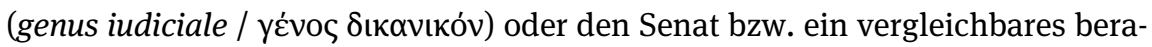

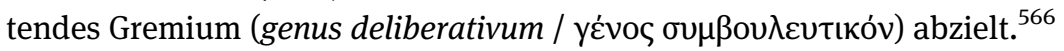

Besonders deutlich geht die erfreuende Wirkung des (Herrscher-)Lobes aus einer Stelle der ersten Suasorie des älteren Seneca hervor, in der Alexander der Große überlegt, ob er den Ozean durchqueren soll (deliberat Alexander an Oceanum naviget), und die Deklamatoren Alexander von diesem Vorhaben abraten. An dieser Stelle referiert Seneca d.Ä. die Instruktionen des Rhetors Cestius, der Anweisungen dazu erteilt, wie man in dem vorliegenden Spezialfall, wenn man einem Alleinherrscher einen Rat gibt, vorgehen soll: ${ }^{567}$

aiebat Cestius hoc genus suasoriarum aliter declamandum esse quam suadendum. non eodem modo in libera civitate dicendam sententiam quo apud reges, quibus etiam quae prosunt ita tamen, ut delectent, suadenda sunt.

[Cestius sagte, dass man diese Art der Suasorien anders deklamieren muss, als dass man einen normalen Ratschlag erteilt. In einer freien Bürgerschaft solle man seine Meinung nicht auf dieselbe Weise äußern wie vor Königen, denen zwar auch dasjenige, was nutzt, geraten werden muss, aber so, dass es erfreut.]

Cestius äußert an dieser Stelle die Anweisung, dass man, wenn man Personen wie Alexander dem Großen etwas rät, nicht nur Nützliches raten soll, sondern seine Ratschläge mit Herrscherlob verknüpfen soll.

Lukian diskutiert das Lob in der Geschichtsschreibung, das die Rezipienten erfreut, im Zusammenhang mit Reflexionen über den Nutzen, der aus der wahren Erzählung resultiert. Daher werden diese Überlegungen in einem anderen Kapitel präsentiert (s. Kap. 4.2.1).

\subsubsection{Gesang}

Dass auch der Gesang Unterhaltung bewirken kann, zeigt sich u. a. an einer Stelle der Odyssee. Über den Sänger Demodokos heißt es nämlich am Anfang des achten Buches, dass er seine Zuhörer erfreut: ${ }^{568}$

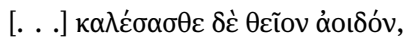

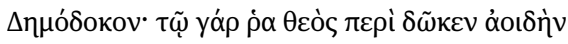

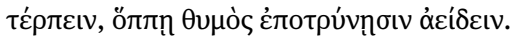

566 Vgl. Cic. part. 10; s. Kap. 1.3. Zur Unterhaltung durch das genus demonstrativum vgl. auch Cic. de orat. 2,341; Quint. inst. 2,10,11; 3,4,6 und 3,8,15. Zu Lob und Kritik s. auch Kap. 2.2.2. 567 Sen. suas. 1,5. Vgl. auch Sen. suas. 1,6 und 8. Zu Text und Kommentar vgl. Feddern (2013). 568 Hom. Od. 8,43-45. 
[[. . . ] ruft den göttlichen Sänger herein, Demodokos; denn ihm ganz besonders hat ein Gott die Gabe des Gesanges verliehen, um zu erfreuen, in welcher Weise auch immer sein Herz ihn zu singen veranlasst.]

Diese Stelle deutet relativ unspezifisch auf die Fähigkeit des Dichters zu singen, wobei der Inhalt des Gesanges ausgeblendet wird. ${ }^{569}$

Mit dem Gesang als Mittel, um die Rezipienten zu erfreuen, ist auch die Lieddichtung ( $\mu \varepsilon \lambda о \pi о$ i $\alpha$ ) vergleichbar, über die in der Aristotelischen Poetik reflektiert wird (s. Kap. 4.1).

\subsection{Nutzen/Belehrung}

Der Nutzen / die Belehrung kann sowohl aus einer wahren als auch aus einer fiktionalen Erzählung resultieren.

\subsubsection{Nutzen/Belehrung durch eine wahre Erzählung}

Vom Nutzen einer wahren Erzählung war bereits die Rede im Zusammenhang mit der Opposition zwischen der Unterhaltung durch eine fiktionale oder eine falsche Erzählung und dem Nutzen / der Belehrung durch einen wahren Bericht (s. Kap. 4.1.1 und Kap. 4.1.2.2). Wenn Thukydides seine eigene historiographische Darstellung von den Produkten der Dichter und Geschichtenerzähler absetzt, zeigt sich in dieser Gegenüberstellung die Auffassung, dass die Geschichtsschreibung vorrangig das Ziel verfolgt, die Rezipienten durch eine wahre Erzählung

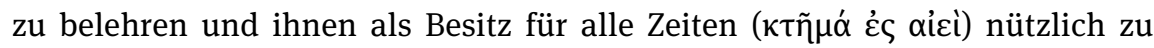
sein (s. Kap. 4.1.2.2).

Auch im Zusammenhang mit Polybios' Kritik an Phylarchos' Darstellung des Kleomenischen Krieges (s. Kap. 2.1.3.3) wird deutlich, dass für die Historiographie insbesondere der Nutzen charakteristisch ist, da Polybios in diesem Kontext die Geschichtsschreibung der Tragödie folgendermaßen gegenüberstellt: ${ }^{570}$

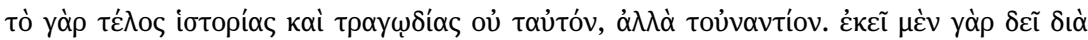

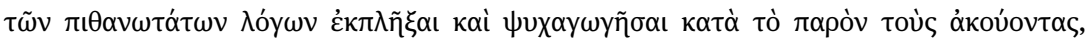

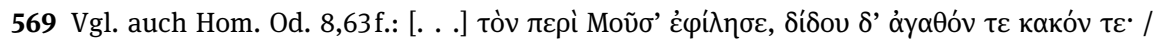

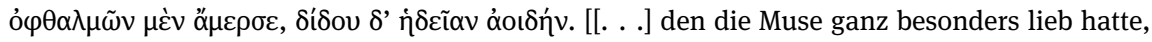
dem sie Gutes und Schlechtes gab; des Augenlichts beraubte sie ihn, sie gab ihm aber den süßen Gesang.]; Hom. Od. 8,266.

570 Polyb. 2,56,11f. 


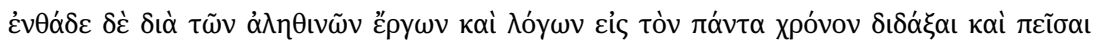

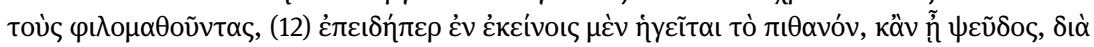

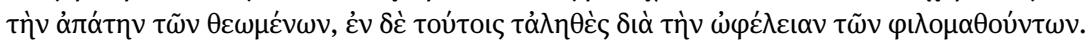

[Denn das Ziel der Geschichtsschreibung und der Tragödie ist nicht dasselbe, sondern entgegengesetzt. Dort muss man nämlich durch die überzeugendsten Worte die Zuhörer für den Augenblick erschüttern und mitreißen, hier durch die wahren Taten und Worte diejenigen die ganze Zeit belehren und überzeugen, die Freude daran haben, etwas zu lernen. (12) Denn in jenem spielt das Überzeugende die Hauptrolle, auch wenn es nicht der Wahrheit entspricht, aufgrund der Täuschung der Zuschauer, in diesem die Wahrheit aufgrund des Nutzens derjenigen, die Freude daran haben, etwas zu lernen.]

Die Geschichtsschreibung ist dadurch nützlich, dass sie die Rezipienten durch einen wahren Bericht belehrt. Andererseits lässt sich an dem Partizip von $\varphi \iota \lambda о \mu \alpha \theta \varepsilon \dot{\varepsilon} \omega$ [Freude daran haben, etwas zu lernen] erkennen, dass die Historiographie den Rezipienten auch Freude bereitet, wobei die Freude ebenso wie der Nutzen aus der Belehrung resultiert (zur Unterhaltung durch eine wahre Erzählung s. Kap. 4.1.2.1).

In ähnlicher Weise, aber - zumindest auf den ersten Blick - noch entschiedener betrachtet Lukian den Nutzen, der aus einer wahren Darstellung resultiert, als das Ziel der Geschichtsschreibung: ${ }^{571}$

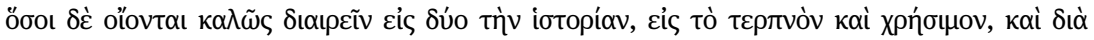

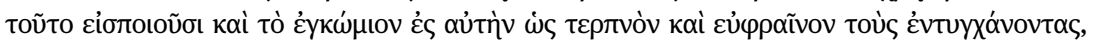

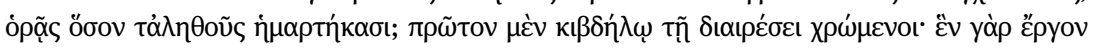

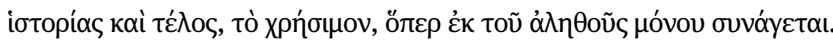

[All diejenigen, die meinen, die Geschichtsschreibung schön zweizuteilen, nämlich in das Erfreuende und das Nützliche, und deswegen auch das Lob in sie integrieren, da es die Rezipienten erfreue und ergötze - siehst du, wie sehr sie sich an der Wahrheit vergangen haben? Erstens tun sie schlecht daran, die Unterteilung zu benutzen. Es gibt nämlich (nur) eine Aufgabe und ein Ziel der Geschichtsschreibung: das Nützliche, was nur aus der Wahrheit resultiert.]

Diese Stelle erweckt den Eindruck, als würde Lukian das Ziel der Geschichtsschreibung auf den aus der Wahrheit resultierenden Nutzen reduzieren und eine unterhaltende Wirkung strikt ausschließen. Seine weiteren Instruktionen

571 Luk. hist. conscr. 9. Zum Lob in der Panegyrik und Dichtung vgl. Luk. hist. conscr. 7-9 (s. Kap. 2.2.2). Zu den beiden Zielen des Nutzens und der Unterhaltung bei Lukian und anderen Historikern vgl. auch Luk. hist. conscr. 42, wo Lukian das Thukydideische Methodenkapitel paraphrasiert; Avenarius (1956, 22-29). 
zeigen aber, dass er keinen ausschließenden Gegensatz zur unterhaltenden Wirkung herstellt: ${ }^{572}$

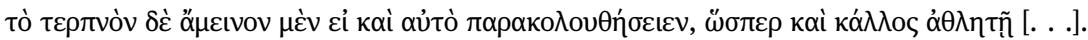

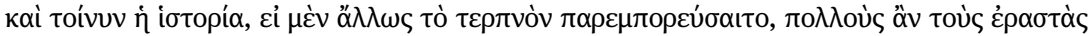

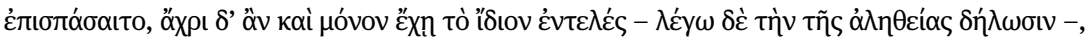

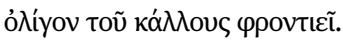

[Wenn auch das Angenehme noch hinzukommt, ist es besser - wie Schönheit zum Athleten [. . .]. Und die Geschichtsschreibung würde, wenn sie zusätzlich auch noch Vergnügen bereiten sollte, gewiss viele Liebhaber anziehen; solange sie aber nur dasjenige vollbringt, was für sie charakteristisch ist - ich meine das Offenlegen der Wahrheit -, wird sie sich wenig um die Schönheit kümmern.]

Der aus der Wahrheit resultierende Nutzen ist also ein notwendiges Ziel der Geschichtsschreibung, wohingegen die - wahrscheinlich aus dem maßvollen Lob (s. Kap. 2.2.2 und 4.1.5), vielleicht auch aus der Stilisierung (s. Kap. 4.1.3) herrührende - Unterhaltung eine optionale Wirkung darstellt.

\subsubsection{Nutzen/Belehrung durch eine fiktionale Erzählung}

Auch eine fiktionale Erzählung kann nach antiker Anschauung die Rezipienten belehren bzw. ihnen nutzen, wobei nicht die wahren Elemente innerhalb der fiktionalen Erzählung, sondern die fiktiven Elemente diese Wirkung hervorrufen. Der Nutzen / die Belehrung durch eine fiktionale Erzählung ist nicht einmal auf eine allegorische fiktionale Erzählung wie die Fabel beschränkt. ${ }^{573}$

\subsubsection{Nutzen/Belehrung durch eine nicht-allegorische fiktionale Erzählung} An einer Stelle der Dichterkritik innerhalb der Politeia (s. Kap. 1.4.1.1), an der der Platonische Sokrates die Unwahrheit in Reden behandelt, nachdem

572 Luk. hist. conscr. 9.

573 Der Sonderfall der faktualen allegorischen Erzählung (der Bibel) kann unberücksichtigt bleiben, da sie zur Kategorie des Nutzens / der Belehrung durch eine wahre Erzählung gehört (s. Kap. 4.2.1). Zur Unterscheidung zwischen der allegoria in verbis und der allegoria in facto vgl. Augustinus' Unterscheidung (Aug. trin. 15,15) zwischen der paganen Allegorie, bei der auf der Oberfläche eine fiktive Geschichte geschildert wird (allegoria in verbis), und der biblischen Allegorie, bei der ein wirkliches Ereignis (oder Sache oder Person), das zumeist im Alten Testament erwähnt wird, ein anderes wirkliches Ereignis bezeichnet, vielleicht sogar präfiguriert, das zumeist im Neuen Testament geschildert wird (allegoria in facto). 
er zwischen der Unwahrheit in der Seele und der Unwahrheit in Reden unterschieden hat, findet sich die folgende Reflexion über den Nutzen von fiktionalen Erzählungen: ${ }^{574}$

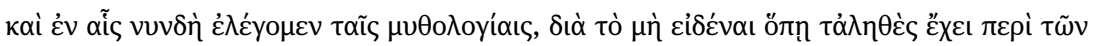

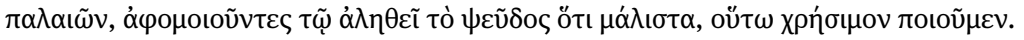

[Das gilt auch für die Geschichten, die wir soeben erwähnt haben: Da wir nicht wissen, wie sich die alten Dinge in Wahrheit verhalten, machen wir das Unwahre nützlich, indem wir es so weit wie möglich an die Wahrheit angleichen.]

Mit den erwähnten Geschichten bezieht sich der Platonische Sokrates v. a. auf die dichterische Darstellung der Götter und Heroen, die seinem Axiom folgen muss, dass die Götter immer gut sind, immer gut handeln und folglich nur Ursache für das Gute sind. ${ }^{575}$ Diese Stelle zeigt, dass der Platonische Sokrates von den Dichtern im Fall von weit zurückliegenden Ereignissen keine strenge Historizität, sondern eine realistische Darstellung fordert, die die Menschen belehrt.

Eine andere Stelle in der Platonischen Dichterkritik zeigt ebenso, dass auch fiktionale Darstellungen dem Zweck des Nutzens dienen können: ${ }^{576}$

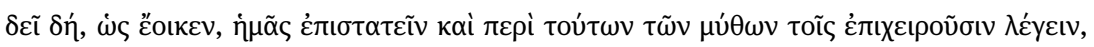

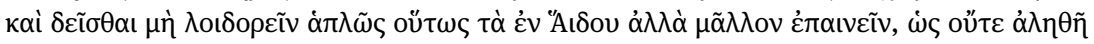

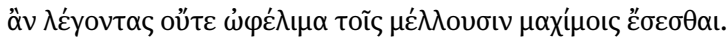

[Wir müssen also, wie es scheint, auch diejenigen kontrollieren, die über diese Geschichten [sc. die Unterwelt] sprechen wollen, und sie bitten, die Zustände im Hades nicht einfach so zu schmähen, sondern vielmehr zu loben, da sie weder die Wahrheit sagen noch etwas, was denjenigen nützt, die für den Kampf geeignet sein sollen.]

Denn die Formulierung des Platonischen Sokrates, dass die Dichter und $\mathrm{Au}$ toren über die Unterwelt weder wahre noch nützliche Geschichten erzählen, lässt die Möglichkeit durchscheinen, eine fiktive Geschichte zu erzählen, die für die Ausbildung der Krieger nützlich und geeignet ist, indem sie zu Tapferkeit ermutigt.

574 Plat. rep. 382d1-4. Zum Unterschied zwischen der Unwahrheit in der Seele und der Unwahrheit in Reden vgl. Plat. rep. 382b-c; Baima (2017).

575 Vgl. Plat. rep. 379a5-379c8.

576 Plat. rep. 386b-c. 
Auch mit Blick auf die Komödie lässt sich in den spätantiken Dramentraktaten die Auffassung antreffen, dass sie nicht nur die Rezipienten unterhält, ${ }^{577}$ sondern sie auch lebenspraktisch belehrt: ${ }^{578}$

comoedia est fabula diversa instituta continens affectuum civilium ac privatorum, quibus discitur, quid sit in vita utile, quid contra evitandum.

[Die Komödie ist ein Bühnenstück, das unterschiedliche, gewohnheitsmäßige Affekte von Bürgern und privaten Personen enthält, durch die man lernt, was im Leben nützlich ist und was man hingegen meiden muss.]

[. . . ] in tragoedia fugienda vita, in comoedia capessenda exprimitur.

[[. . .] in der Tragödie wird dasjenige Leben dargestellt, das man meiden soll, in der Komödie dasjenige, das man ergreifen soll.]

Der belehrende Effekt der Komödie bzw. des Dramas wird sich auf den Handlungsverlauf und v. a. das Ende des Dramas beziehen, also in der Komödie auf das happy end. ${ }^{579}$

\subsubsection{Nutzen/Belehrung durch eine allegorische fiktionale Erzählung}

Über den Nutzen / die Belehrung durch die Fabel, also durch eine allegorische fiktionale Erzählung, reflektiert u. a. (Pseudo-)Hermogenes in seinem Progymnasmata-Handbuch:: 580

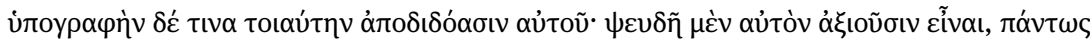

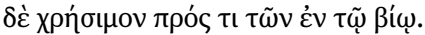

577 Zur unterhaltenden Funktion der Komödie vgl. Macr. somn. 1,2,7f.: fabulae, quarum nomen indicat falsi professionem, aut tantum conciliandae auribus voluptatis, aut adhortationis quoque in bonam frugem gratia repertae sunt. auditum mulcent [. . .] comoediae, quales Menander eiusve imitatores agendas dederunt [. . .]. [Die fiktiven Geschichten (fabulae), deren Name die Verkündung von Unwahrem anzeigt, sind entweder erfunden worden, um nur den Ohren Vergnügen zu bereiten, oder auch, um zur Besserung aufzufordern. Die Ohren ergötzen [. . .] Komödien, wie sie Menander oder dessen Nachahmer haben aufführen lassen [. . .].]; Euanthius, De fabula 2,6; Isid. orig. 1,40,3. Zu Macrobius' Untergliederung der fabula vgl. Feddern (2018, 379-383).

578 Ael. Don. com. V 1; Euanthius, De fabula 4,2. Zu den Dramentraktaten vgl. die Textausgabe von Koster (1975). Zu Euanthius, De fabula vgl. die Ausgabe mit Kommentar von Cupaiuolo (1992). Zur lateinischen Dramentheorie und zu den dramatischen Gattungen vgl. Manuwald (2016, 101-137).

579 Zur Diskussion über das happy end in der Komödie von der Antike bis in das zwanzigste Jahrhundert vgl. Kraft (2011).

580 Hermog. Progym. 1,4 Patillon (2008, 181). 
[Eine derartige skizzenhafte Beschreibung gibt man von ihr [sc. der Fabel]: Man ist der Auffassung, dass sie fiktional, aber auf jeden Fall für irgendeinen Aspekt des Lebens nützlich sein soll.]

Dabei dürfte evident sein, dass sich der Nutzen auf die allgemeine, zumeist moralische Wahrheit bezieht, die die allegorische Fabel transportiert. ${ }^{581}$

\section{3 Überzeugung/Plausibilität}

Bekanntermaßen ist die Überredung bzw. Überzeugung das Ziel der rhetorischen Rede, wie an vielen Stellen der Rhetorik-Handbücher festgestellt wird, u. a. in Ciceros De inventione: ${ }^{582}$

officium [. . . ] eius [sc. oratoriae] facultatis videtur esse dicere adposite ad persuasionem; finis persuadere dictione.

[Die Aufgabe dieser [sc. rhetorischen] Kompetenz scheint zu sein, angemessen zu reden, um zu überzeugen; das Ziel ist, durch die Rede zu überzeugen.]

581 Denn die Fabel ( $\mu$ ṽ o૬) wird traditionellerweise so definiert, dass sie eine fiktionale Erzäh-

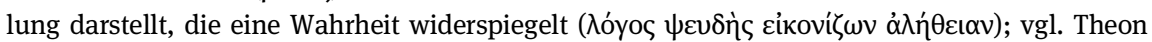
RhG II 72,28 Spengel (Patillon und Bolognesi 1997, 30); Aphth. Progym. 1,1 Patillon (2008, 112); Nikolaos RhG XI 6,9 Felten; Priscian, Praeexercitamina Passalacqua (1987, 33): fabula est oratio ficta [. . .] imaginem exhibens veritatis. Zum nützlichen Aspekt der Fabel durch eine moralische

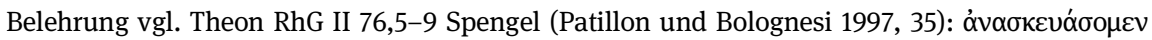

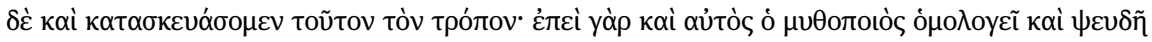

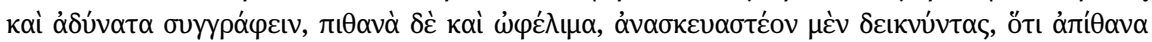

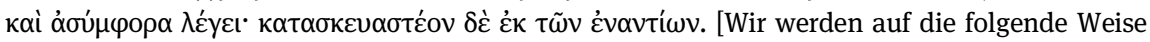
die Widerlegung und die Bekräftigung anstellen: Da ja der Fabelschreiber selbst eingesteht, dass er Unwahres und Unmögliches, aber Glaubwürdiges und Nützliches verfasst, muss man die Widerlegung anstellen, indem man zeigt, dass er Unglaubwürdiges und Schädliches sagt; die Bekräftigung muss man in umgekehrter Weise durchführen.]; Hermog. Progym. 1,1 Patillon

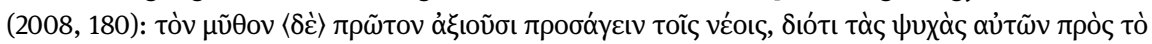

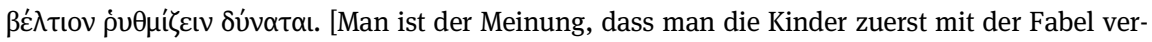
traut machen muss, weil sie ihre Seele zum Besseren bilden kann.]

582 Cic. inv. 1,6. Etwas anders definiert Aristoteles die Aufgabe und das Ziel der Rhetorik, da er das Erblicken des Überzeugenden und nicht die Überzeugung selbst als Ziel betrachtet;

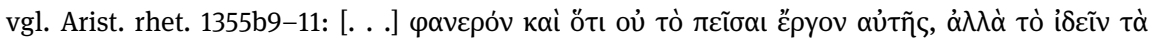

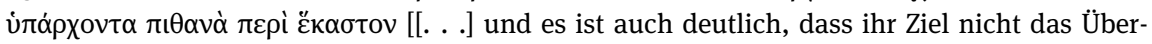
zeugen ist, sondern das vorliegende Überzeugende in jedem Einzelfall zu erblicken.]; Arist.

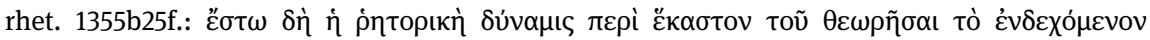
$\pi \imath$ avóv [Die Rhetorik sei verstanden als Fähigkeit, in jedem Einzelfall das möglicherweise Überzeugende zu erblicken.] 
Dabei ist das Ziel der Überzeugung primär funktional zu sehen, da der Redner zumindest in den ersten beiden genera dicendi, der Gerichtsrede (genus iudi-

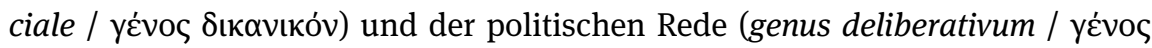

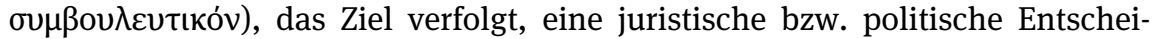
dung herbeizuführen, indem er die Richter $z u$ einem bestimmten Urteil bzw. das beratende Gremium wie den Senat zu einem bestimmten Entschluss veranlasst (s. Kap. 1.3). Im Fall der Prunkrede (dem genus demonstrativum / yévos

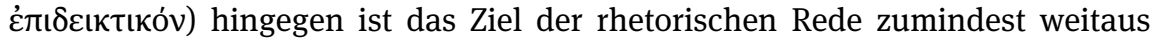
weniger funktional, da der Redner eine andere Person lobt oder kritisiert.

Die Überzeugung ist aber auch ein Ziel vieler anderer erzählender Gattungen, und zwar unabhängig von ihrer möglichen Funktionalität und ihrer Faktualität bzw. Fiktionalität. Schon in den Rhetorik-Handbüchern lässt sich erkennen, dass die Überzeugung zwar insbesondere ein Kriterium der Erzählung (narratio) ist, dass die Instruktionen zur Erzählung aber nicht nur für die Schilderung des Tathergangs vor Gericht relevant sind, sondern auch für die anderen Teile der Rede gelten und über die Rede hinausweisen. ${ }^{583}$ Daher können die Instruktionen zur plausiblen Erzählung des Auctor ad Herennium als Anweisungen für alle erzählenden Gattungen gelesen werden (s. Kap. 2.1.3.2). Im Unterschied zur Rhetorik, in der das Überzeugen vom eigenen Standpunkt das Ziel und die Plausibilität das Mittel ist, stellt für die anderen erzählenden Gattungen eher die Plausibilität selbst, das eigene (poetische) Können, das Ziel dar.

In Aristoteles' Reflexionen über den Gegenstandsbereich und Realitätsbezug der Tragödie lässt sich erkennen, dass die Tragödie auch das Ziel verfolgt, die Zuschauer zu überzeugen. Denn Aristoteles sieht die Aufgabe des Dichters darin, das Mögliche darzustellen, worunter er das Geschehene und die realistische Fiktion versteht, und begründet die Behandlung von historischen Stoffen damit, dass das Mögliche, wozu das Geschehene gehört, überzeugend ist (s. Kap. 1.4.1.2).

Wenn Polybios im Kontext seiner Kritik an Phylarchos die Geschichtsschreibung der Tragödie gegenüberstellt, wird aus dieser Opposition deutlich, dass die Ziele beider Gattungen zwar verschieden sind, dass aber eine gemeinsame Wirkung darin besteht, die Rezipienten zu überzeugen (s. Kap. 4.2.1).

Strabo schließt sich Polybios' Ansicht an, dass Homer in seinen Epen wirkliche und erfundene Ereignisse miteinander verknüpft hat (s. Kap. 1.4.1.3), und

583 Vgl. Cic. de orat. 2,83; Cic. part. 31. Die Annahme, dass diese Anforderungen an die Erzählung für alle erzählenden Gattungen gelten, lässt sich dadurch belegen, dass die Erzählung einleitend allgemein als Darstellung von Ereignissen definiert wird und verschiedene Formen der literarischen Erzählung umfasst, die explizit unterschieden werden; s. Kap. 1.1.2 und (zur sog. Skalierung der dargestellten Geschichte) Kap. 1.4.2.2. 
sieht ein Ziel der Vermischung von Fakten und Fiktionen in der überzeugenden Wirkung der Erzählung. Diese Ansicht stützt er mittels eines Verses aus der Odyssee, mit dem Homer diejenige Lügengeschichte kommentiert, die Odysseus seiner Frau Penelope erzählt: ${ }^{584}$

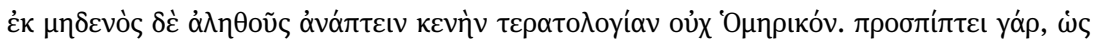

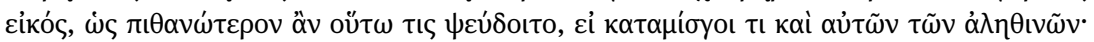

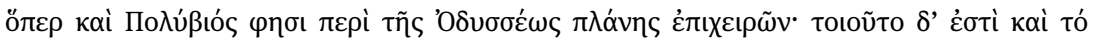

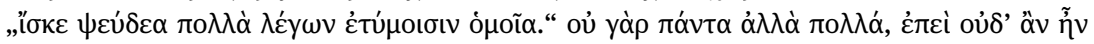

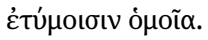

[An nichts Tatsächliches anknüpfend bloß Wunderdinge zu erzählen ist nicht Homers Sache. Stellt sich doch begreiflicherweise unwillkürlich die Überlegung ein, dass jemand auf diese Art überzeugender die Unwahrheit sagen wird, wenn er auch etwas von eben dem Tatsächlichen daruntermischt (was auch Polybios in seiner Erörterung der Irrfahrt des Odysseus sagt). Das bedeutet auch der Vers „Während er sprach, machte er viele falsche Dinge den wahren ähnlich.“ Heißt es doch nicht „alle“, sondern „viele“, da sie sonst nicht den wahren ähnlich wären.]

Dabei versteht Homer die Ähnlichkeit mit der Realität v. a. qualitativ und in Bezug auf eine Lügengeschichte (Odysseus gestaltet plausible Lügengeschichten), während Strabo sie v. a. quantitativ und mit Blick auf die Fiktion versteht (Homer gestaltet eine überzeugende fiktionale Erzählung, indem er nicht nur fingiert, sondern auch viele Realien schildert).

\subsection{Exemplifizierung}

In der modernen Erzähl- und Fiktionstheorie wird darüber diskutiert, ob (fiktionale) Erzählungen die spezifische Funktion erfüllen, eine exemplifizierende Lektüre zu ermöglichen. So unterscheidet Stierle, ausgehend von einem rezeptionsästhetisch orientierten Literaturverständnis, zwischen der quasipragmatischen und der fiktionserfassenden Rezeption fiktionaler Erzählungen, wobei die letztere die Verschiebung der Orientierung von der Ebene der erzählten

584 Strabo 1,2,9. Vgl. Hom. Od. 19,203; im Kontext der Stelle erzählt Odysseus Penelope die Lügengeschichte, dass er Aithon, ein Bruder des Idomeneus, sei. Als Odysseus auf dem Weg nach Troja gewesen sei, habe er ihn in Amnisos auf Kreta freundlich empfangen, da Idomeneus bereits aufgebrochen war; vgl. Hom. Od. 19,165-202. Zu den Trugreden in der Odyssee vgl. Grossardt (1998); Fuchs (1993). 
Geschichte $\mathrm{zu}$ einer konzeptuellen, allgemeineren Ebene bedeutet, worunter Stierle mögliche Organisationsschemata der Erfahrung versteht. ${ }^{585}$

Über die exemplifizierende Funktion von Erzählungen wurde bereits in der Antike diskutiert. Der sicherlich bekannteste Ort ist das neunte Kapitel der Aristotelischen Poetik, in dem Aristoteles die Dichtung als philosophischer und besser als die Geschichtsschreibung bezeichnet, weil die erstere Gattung eher das Allgemeine, die letztere aber das Einzelne sagt (s. Kap. 1.4.1.2). Den Hintergrund für Aristoteles’ Äußerungen über das Einzelne und das Allgemeine bildet seine wissenschaftstheoretische Unterscheidung zwischen dem zufälligen Einzelnen und dem notwendigen Allgemeinen, wobei diese Zweiteilung häufig um

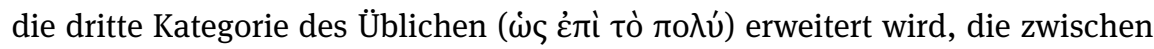
dem Notwendigen und dem Zufälligen liegt, aber dem Notwendigen insofern näher als dem Zufälligen steht, als es ebenso wie das Notwendige volles wissenschaftliches Wissen konstituiert. ${ }^{586}$

Nach Aristoteles' Dichtungskonzeption liegt das Allgemeine in der wahr-

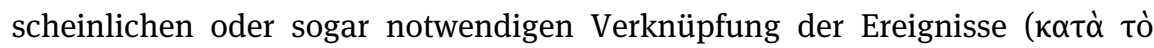

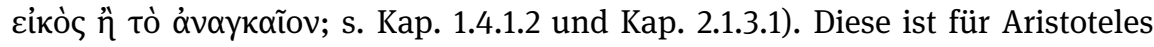
eine notwendige Anforderung an die Dichtung, wobei es nicht ausgeschlossen ist, dass auch in der Geschichtsschreibung eine motivierte Ereignissequenz geschildert wird (s. Kap. 2.1.3.3). Nicht nur beim Gegenstandsbereich, sondern auch in dieser Hinsicht kann es also zu Überschneidungen zwischen der Dichtung und der Geschichtsschreibung kommen. ${ }^{587}$

Im Folgenden sollen weitere antike Reflexionen über die Exemplifizierung analysiert werden. Ihnen ist gemeinsam, dass sie das Einzelne und Allgemeine empirisch und somit anders als Aristoteles auffassen. Darüber hinaus zeigen sie, dass die Exemplifizierung eine Funktion ist, die nach antiker Anschauung sowohl faktuale als auch fiktionale Erzählungen erfüllen.

585 Vgl. Stierle (1975) und Stierle (1983). Stierles Konzept wirft aber die Probleme auf, dass nicht alle fiktionalen Erzählungen eine fiktionserfassende Rezeption ermöglichen, da Trivialromane nicht in dieser Form rezipiert werden können (dieses Problem ließe sich u. U. durch einen engen Literaturbegriff beheben), und dass auch faktuale Texte den Rezipienten die Gelegenheit dazu geben, im Besonderen das Allgemeine zu erblicken; vgl. Zipfel (2001, 262-266); Feddern (2018, 90-94).

586 Vgl. de Sainte Croix (1992, 24-28). Zum Zufälligen vgl. auch Schmitt (2011, 403f.) zum neunten Kapitel der Poetik.

587 Auch der Gegenstandsbereich des Dichters kann sich mit demjenigen des Historikers überschneiden, nämlich dann, wenn der Dichter unter dem Möglichen das Geschehene wählt (der Dichter könnte die Komponente der Möglichkeit auch berücksichtigen, indem er eine mögliche Handlung erfindet; der Historiker muss das Geschehene darstellen); s. Kap. 1.4.1.2. 
Die Auffassung, dass wahre ebenso wie erfundene Beispielerzählungen Verallgemeinerungspotential haben, lässt sich z. B. in Aristoteles' Rhetorik antreffen. Denn Aristoteles betrachtet in Kapitel 2,20 bei der Behandlung der allgemeinen Argumente das Beispiel als induktives Beweismittel neben dem Enthymem und unterscheidet zwischen wahren und erfundenen Beispielen: ${ }^{588}$

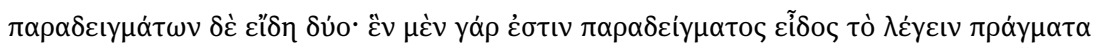

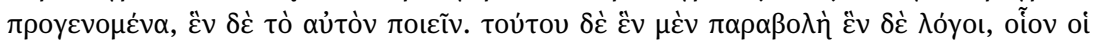

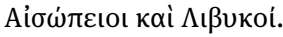

[Es gibt zwei Arten von Beispielen. Eine Art des Beispiels besteht nämlich darin, Ereignisse zu nennen, die vorher stattgefunden haben, eine [sc. andere Art des Beispiels besteht darin], sie selbst zu erfinden. Zu der zuletzt genannten Art gehört zum einen der Vergleich, zum anderen die Fabeln, wie die Äsopischen und Libyschen.]

Funktional besteht also kein Unterschied zwischen den wahren und erfundenen Beispielen. Es besteht aber ein praktischer Unterschied darin, dass die letzteren den Vorteil bieten, sie situationsgerecht zu erfinden und zu verwenden. ${ }^{589}$

Wenn Cicero an einer Stelle seiner Verteidigungsrede für Sextus Roscius beiläufig auf die Fiktionalität der Komödie zu sprechen kommt, zeigt sich auch in diesen Reflexionen die Einsicht, dass die Exemplifizierung eine Funktion ist, die sowohl faktuale als auch fiktionale Erzählungen erfüllen: ${ }^{590}$

ecquid tandem tibi videtur, ut ad fabulas veniamus, senex ille Caecilianus minoris facere Eutychum, filium rusticum, quam illum alterum, Chaerestratum? - nam, ut opinor, hoc nomine est - alterum in urbe secum honoris causa habere, alterum rus supplici causa relegasse? „quid ad istas ineptias abis?“ inquies. [. . .] homines notos sumere odiosum est, cum et illud incertum sit velintne ei sese nominari, et nemo vobis magis notus futurus sit quam

588 Arist. rhet. 1393a28-31. Den Vergleich exemplifiziert Aristoteles anhand des Sokratischen Arguments bzw. Vergleichs, dass nicht diejenigen herrschen sollen, die durch das Los bestimmt wurden. Das sei nämlich so ähnlich, wie wenn man als Athleten durch das Los bestimmen würde nicht diejenigen, die für den Wettkampf geeignet sind, sondern diejenigen, die Losglück haben, oder wenn man einen Seemann, der Losglück hat, zum Steuermann auserwählt und nicht jemanden, der etwas davon versteht; vgl. Arist. rhet. 1393b4-8. Zu Sokrates' Verwendung dieser Parabel vgl. Xen. mem. 1,2,9. Auch bei Cicero (vgl. Cic. inv. 1,49) fungiert das Beispiel (exemplum) als Teil der Argumentation. Vgl. auch Quint. inst. 5,11,6 und 19, der darauf hinweist, dass Beispiele fiktiv sein können, wodurch er sie analog zur Erzählung definiert (vgl. Quint. inst. 4,2,31), und die Fabel als eine Art des Beispiels bespricht. In der Herennius-Rhetorik hingegen wird das Beispiel als historisches Beispiel definiert; vgl. rhet. Her. 4,62.

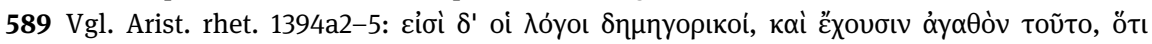

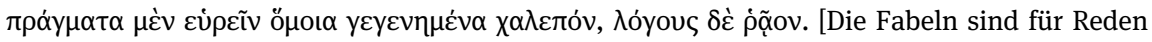
vor dem Volk geeignet und haben den folgenden Vorteil, dass es schwierig ist, ähnliche Ereignisse $\mathrm{zu}$ finden, die geschehen sind, aber einfacher ist, Fabeln zu erfinden.]

590 Cic. S. Rosc. 46 f. 
est hic Eutychus, et certe ad rem nihil intersit utrum hunc ego comicum adulescentem an aliquem ex agro Veienti nominem. etenim haec conficta arbitror esse a poetis ut effictos nostros mores in alienis personis expressamque imaginem vitae cotidianae videremus.

[Scheint dir etwa, um zu (fiktiven) Geschichten zu kommen, jener alte Mann bei Caecilius den Eutychus, seinen ländlichen Sohn, weniger zu mögen als jenen anderen, Chaerestratus - denn das ist, wie ich glaube, sein Name - und den einen ehrenhalber in der Stadt bei sich zu haben, den anderen auf das Land verbannt zu haben, um ihn zu bestrafen? „Warum schweifst du zu diesen Albernheiten ab?“, wirst du fragen. [. . .] Man zieht Hass auf sich, wenn man bekannte Menschen heranzieht, weil auch dies unsicher ist, ob sie genannt werden wollen, und niemand euch bekannter sein wird, als es dieser Eutychus ist, und es sicherlich egal ist, ob ich diesen jungen Mann aus der Komödie oder irgendjemanden aus dem ländlichen Gebiet von Veii nenne. Denn ich glaube, dass dies von den Dichtern erfunden worden ist, damit wir einen Ausdruck unserer Lebensweise in fremden Figuren und ein anschauliches Bild des täglichen Lebens sehen.]

Möglicherweise geht Ciceros berühmtes Diktum, dass die Komödie ein Spiegel des Lebens ist, auf diese Stelle zurück. ${ }^{591}$ Cicero drückt in einer rhetorischen Frage den Gedanken aus, dass es keine Bestrafung ist, wenn ein junger Mann auf dem Land aufwächst, und bekräftigt diesen Gedanken mit dem Beispiel des Eutychus, einer Figur, die wohl aus Caecilius' Komödie Hypobolimaeus stammt. ${ }^{592}$ Er antizipiert den möglichen Einwand, dass sein Beispiel ungültig sei, da es der fiktionalen Gattung der Komödie entstamme, durch seine Einschätzung, dass die Komödie das alltägliche Leben der Menschen nachahmt. Diese fiktionstheoretische Einschätzung resultiert zum einen aus dem mimetischen Charakter der Dichtung und zum anderen aus der Zuordnung der Komödie zur Erzählgattung argumentum, also zur realistischen Fiktion, da die Handlung der Komödie insgesamt gesehen erfunden ist, ihre einzelnen Elemente aber aus der Alltagswirklichkeit herrühren (s. Kap. 1.4.2.2).

Die Funktion der Fiktion besteht in dem Verallgemeinerungspotential: Da die Komödie Ereignisse schildert, die so oder so ähnlich jederzeit geschehen (könnten), spiegelt sie menschliches Verhalten wider, das sich in der fiktiven Welt der Komödie nicht (oder kaum) anders zeigt als in der Realität.

591 Vgl. Ael. Don. com. V 1: comoediam esse Cicero ait imitationem vitae, speculum consuetudinis, imaginem veritatis. [. . .] aitque esse comoediam cotidianae vitae speculum [. . .]. [Die Komödie, sagt Cicero, ist Nachahmung des Lebens, Spiegel der Gewohnheit, Abbild der Wirklichkeit. [. . . ] Und er [sc. Cicero] sagt, die Komödie sei ein Spiegel des täglichen Lebens [. . . ].] Andere mögliche und überlieferte Kontexte sind Cic. rep. 4,20a Powell (= Aug. civ. 2,9) und Hortens. fr. 10 Grilli. Die Ansicht, dass die Komödie ein Spiegel des menschlichen Lebens ist, hat einen weiteren Vorläufer in Theophrast (fr. 708 Fortenbaugh), der den Mimos als Mimesis des Lebens bezeichnet hat. Zu Donats Komödientraktat s. Kap. 4.2.2.1.

592 Vgl. die Kommentare von Dyck $(2010,116)$ und Landgraf $(1914,105-108)$ zu Cic. S. Rosc. $46 f$. 
Insofern entsprechen Ciceros Reflexionen über die Fiktionalität der Komödie teilweise denjenigen des Aristoteles, der die Dichtung als Mimesis betrachtet (s. Kap. 1.1.3.2) und gerade in der Komödie den Allgemeinheitscharakter der Dichtung erblickt (s. Kap. 1.4.1.2). Allerdings sieht Aristoteles das Allgemeine in der Handlungslogik, wohingegen Cicero auf das empirisch Allgemeine abzielt. Im Verallgemeinerungspotential sieht Cicero aber keine Wirkung bzw. Funktion, die einen fiktionalen Text von einem faktualen Text unterscheidet, da es, wie Cicero treffend feststellt, egal ist, ob man eine bekannte Figur aus der Komödie oder irgendjemanden aus dem ländlichen Gebiet von Veii als Beispiel für eine Person nennt, die ein glückliches Leben auf dem Land führt.

Weitere Belege für die Ansicht, dass wahre ebenso wie erfundene Beispielerzählungen Verallgemeinerungspotential haben, ließen sich beliebig hinzufügen. ${ }^{593}$ So bespricht Horaz in einem Brief, inwiefern Homers Epen eine in ethischer Hinsicht exemplifizierende Lektüre ermöglichen. ${ }^{594}$ Die Ilias schildert ihm zufolge durch ihre individuelle Handlung die folgende allgemeine Einsicht: ${ }^{595}$

fabula, qua Paridis propter narratur amorem

Graecia barbariae lento conlisa duello,

593 Ovid äußert in einem Brief vom Schwarzen Meer, der an den Dichter Albinovanus Pedo adressiert ist, die Ansicht, dass jeder ein Theseus in der Hinsicht sein kann, dass er seinem bedrängten Freund zur Seite steht; vgl. Ov. Pont. 4,10,78: in [. . .] fide Theseus quilibet esse potest [in der Treue kann ein jeder ein Theseus sein]. Theseus war u. a. für seine Freundschaft zu Pirithous bekannt, die sich auch darin niederschlug, dass der Held seinen Freund in die Unterwelt begleitete, als dieser beabsichtigte, Persephone zu heiraten (vgl. Ov. trist. 1,5,19f.). Aus dieser offensichtlichen Fiktion gewinnt der ins Exil verbannte Ovid die Einsicht, dass jeder Mensch ein Theseus sein kann, also seinem auf Hilfe angewiesenen Freund helfen kann. Bei Ovid lässt sich aber gleichzeitig erkennen, dass er auch in als historisch betrachteten Personen und Ereignissen Beispiele für die Freundschaft erblickt. In diesem Zusammenhang muss man sich vor Augen führen, dass die meisten Figuren und Geschichten, die wir als Mythen bezeichnen, in der Antike im Kern als historische Personen und Geschichten aufgefasst wurden (s. Kap. 1.4.2.2). Daher beschließt Ovid an einer anderen Stelle eine Beispielreihe für vorbildliche Freundschaft, zu der auch Theseus gehört, mit der Aussage, dass es sich hierbei um Beispiele aus der grauen Vorzeit (exempla priorum [Beispiele früherer [sc. Menschen]]) handelt; vgl. Ov. trist. 1,5,31f. Zu Ov. Pont. 4,10 vgl. Davisson (1982). Zu mythologischen Beispielen in Ovids Exildichtung vgl. Davisson (1993). Zur Mythologie in Ovids Exildichtung vgl. Schubert (1992).

594 Vgl. Hor. epist. 1,2,3f.: [. . .] qui, quid sit pulchrum, quid turpe, quid utile, quid non, / plenius ac melius Chrysippo et Crantore dicit. [[. . . ] der [sc. Homer] ausführlicher und besser als Chrysipp und Crantor sagt, was schön ist, was moralisch schlecht, was nützlich, was es nicht ist.] Zum Brief 1,2 vgl. Manzoni (2015); Hunter (2014); Keane (2010-2011). Zu Horaz’ Ars poetica und den Briefen insgesamt s. Kap. 3.1.1.

595 Hor. epist. 1,2,6-16. 
stultorum regum et populorum continet aestum.

Antenor censet belli praecidere causam;

quid Paris? ut salvus regnet vivatque beatus

cogi posse negat. Nestor componere litis

inter Pelidem festinat et inter Atriden;

hunc amor, ira quidem communiter urit utrumque.

quicquid delirant reges, plectuntur Achivi.

seditione, dolis, scelere atque libidine et ira

Iliacos intra muros peccatur et extra.

[Die Geschichte, in der von Griechenland erzählt wird, das wegen Paris' Liebe in einem langsamen Krieg mit dem Barbarenland zusammengestoßen ist, enthält das hitzige Verhalten von dummen Königen und Völkern. Antenor beabsichtigt, den Grund für den Krieg zu nehmen; was macht Paris? Um wohlbehalten $\mathrm{zu}$ herrschen und glücklich $\mathrm{zu}$ leben, sagt er, dass er nicht [sc. zur Übergabe der Helena] gezwungen werden kann. Nestor bemüht sich darum, schnell den Streit zwischen dem Peliden und dem Atriden beizulegen; dieser brennt vor Liebe, zumindest vor Wut brennt der eine ebenso wie der andere. Was auch immer die Könige in ihrem Wahn tun, darunter müssen die Griechen leiden. Durch Aufruhr, Arglist, Verbrechen, Lust und Zorn werden innerhalb und außerhalb der Trojanischen Mauern Fehler begangen.]

Horaz zufolge darf die Ilias also weitgehend als Negativbeispiel gelesen werden, wie man sich nicht verhalten sollte, wobei das Verhalten von Paris, der Helena entführt hat, und insbesondere dasjenige der beiden Streithähne Achill und Agamemnon kritisch beleuchtet wird. ${ }^{596}$

In der Odyssee erblickt Horaz hingegen die Schilderung des vorbildlichen Verhaltens des Helden: ${ }^{597}$

rursus, quid virtus et quid sapientia possit, utile proposuit nobis exemplar Vlixen, qui domitor Troiae multorum providus urbes, et mores hominum inspexit, latumque per aequor, dum sibi, dum sociis reditum parat, aspera multa pertulit, adversis rerum inmersabilis undis. Sirenum voces et Circae pocula nosti; quae si cum sociis stultus cupidusque bibisset, sub domina meretrice fuisset turpis et excors, vixisset canis inmundus vel amica luto sus. nos numerus sumus et fruges consumere nati, sponsi Penelopae nebulones Alcinoique in cute curanda plus aequo operata iuventus,

596 Zum Verhalten von Achill und Agamemnon vgl. Plut. mor. 26b-f (s. Kap. 2.1.5.2).

597 Hor. epist. 1,2,17-31. 
cui pulchrum fuit in medios dormire dies et

ad strepitum citharae cessatum ducere curam.

[Was auf der anderen Seite die Tugend und was die Weisheit vermag, dafür hat er uns als nützliches Vorbild Odysseus vor Augen gestellt, der als Bezwinger von Troja vorsichtig die Städte und die Verhaltensweise von vielen Menschen betrachtet hat und auf dem weiten Meer, während er für sich und seine Gefährten die Rückkehr bereitet hat, viel Mühsal ertragen hat, ohne in den widrigen Wogen der Dinge unterzugehen. Du kennst die Stimmen der Sirenen und die Becher der Cirke; wenn er aus ihnen dumm und begierig mit den Gefährten getrunken hätte, wäre er unter einer Lustdame und Gebieterin schimpflich und ohne Verstand gewesen und hätte als schmutziger Hund oder als Sau, die den Dreck mag, gelebt. Wir sind eine Nummer und dazu geboren, Früchte zu verzehren, Taugenichtse, die Penelope versprochen wurden, und die jungen Leute des Alcinoos, die mit der Hautpflege über Gebühr beschäftigt waren und für die es schön war, bis zur Mitte des Tages zu schlafen und beim Klang der Zither die Sorgen ruhen zu lassen.]

Im Gegensatz zu seinen Gefährten hat Odysseus also in vorbildlicher Weise Klugheit und Tugend bewiesen, indem er sich nicht vom Gesang der Sirenen hat anlocken lassen und nicht vom Trank der Zauberin Circe gekostet hat, durch den die Gefährten in Schweine verwandelt wurden. ${ }^{598}$

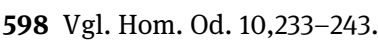


